\title{
Waveform Analysis and Optimization for Radar Coincidence Imaging with Modeling Error
}

\author{
Xiaoli Zhou, Hongqiang Wang, Yongqiang Cheng, Yuliang Qin, and Haowen Chen \\ School of Electronic Science and Engineering, National University of Defense Technology, Changsha 410073, China \\ Correspondence should be addressed to Xiaoli Zhou; zhouxiaoli@nudt.edu.cn
}

Received 27 October 2016; Accepted 20 February 2017; Published 5 March 2017

Academic Editor: Raffaele Solimene

Copyright ( 2017 Xiaoli Zhou et al. This is an open access article distributed under the Creative Commons Attribution License, which permits unrestricted use, distribution, and reproduction in any medium, provided the original work is properly cited.

\begin{abstract}
RCI is a novel superresolution staring imaging technique based on the idea of wavefront modulation and temporal-spatial stochastic radiation field. For RCI, the reference matrix should be known accurately, and the imaging performance depends on the incoherence property of the reference matrix. Unfortunately, the modeling error, which degrades the performance significantly, exists generally. In this paper, RCI using frequency-hopping waveforms (FH-RCI) is considered, and a FH code design method aiming to increase the robustness of RCI to modeling error is proposed. First, we derive the upper bound of imaging error for RCI with modeling error and conclude that the condition number of the reference matrix determines the imaging performance. Then the object function for waveform design which minimizes the condition number of the reference matrix is achieved, and the quantum simulated annealing (QSA) is employed to optimize the FH code. Numerical simulations show that the optimized FH code could decrease the condition number of the reference matrix and improve the imaging performance of RCI with modeling error.
\end{abstract}

\section{Introduction}

Radar coincidence imaging (RCI) is a novel staring imaging technique without the limitation of the target relative motion $[1,2]$. Originated from the optical coincidence imaging, RCI imitates the randomly fluctuant optical-field by constructing temporal-spatial stochastic radiation field in the imaging area. Then focused high-resolution imaging and instantaneous imaging can be achieved by RCI; other significant potentials include interference and jamming suppression. Thus RCI can be used in some important applications, for example, high-resolution earth observation on the static/quasi-stationary platform and disaster monitoring.

For RCI, its essential principle is to form the temporalspatial stochastic radiation field by transmitting stochastic waveforms, while the frequency-hopping $(\mathrm{FH})$ waveforms are good candidates because they are easily generated and have constant modulus $[3,4]$. Besides, FH waveforms can suppress the range ambiguity, decouple the range and Doppler [5], and give immunity from interference with low probabilities of interception and detection. Thus, we consider $\mathrm{RCI}$ using $\mathrm{FH}$ waveforms (FH-RCI).
RCI can be formed as a linear inverse problem which reconstructs the scattering coefficients from the reference matrix and the measurements. Solving such a problem strongly depends on the perfect incoherence property of the reference matrix, while the reference matrix is closely related with the transmitted waveforms. Thus designing an optimal waveform could be helpful in forming a well-structured reference matrix. Various studies have been presented on waveform design. Most of these methods concentrate on radar ambiguity function based design $[3,6-8]$ and covariance matrix based design [9-11], where the waveform is optimized to have a good autocorrelation or cross-correlation property sharpening the ambiguity function or optimizing the waveform covariance matrix. Applying information theoretic to waveform design, two optimal waveforms, based on mutual information maximization and Kullback-Leibler divergence maximization, respectively, are studied for MIMO radar target recognition [12]. Benefiting from a significantly lower computational complexity as compared to an ambiguity-based design method, a cost function based on the hit-matrix is used to design the FH code for MIMO radar [13]. 
In recent years, sparse recovery and compressive sensing (CS) have been a hot topic and applied to radar imaging including RCI, by considering the sparse prior of target [2, 14-16]. The sparse recovery accuracy is determined by the correlations between the columns of the dictionary matrix [4]; thus minimizing the coherence measure ensures theoretical guarantee for sparse support recovery of signals with potentially higher sparsity level. Based on this conclusion, the CS radar waveform design was investigated by minimizing the cross correlations between different target responses [17]. Similarity, Subotic examined the waveform and position impacts by considering the sparsity of the target scene and the restricted isometry property (RIP) [18]. In [19, 20], the transmission waveform and sensing matrix are optimized by minimizing the difference between the Gram matrix of sensing matrix and the identity matrix. To minimize the Cramér-Rao bound (CRB) of sparse recovery, an adaptive waveform design approach was presented to design the carrier frequencies [5]. Based on the sparse recovery model, Gogineni considered the optimal waveform design of MIMO radar by reducing the block coherence measure of the sensing matrix and selecting the FH codes of all the transmitters [4]. Comparably, Han proposed a joint optimization algorithm using game theory to compute the $\mathrm{FH}$ code matrix and amplitude matrix for $\mathrm{FH}$ waveform, based on two objective functions derived by using the ambiguity function and sparse recovery [21].

In this paper, we focus on the waveform design (more precisely, FH code design) for FH-RCI with modeling error, since the modeling error generally exists, for example, gainphase error [2, 14], off-grid error $[15,16,22]$, and array position error [23]. Modeling error would destroy the ideal assumption that the reference matrix is accurately known; thus the performance of RCI degrades significantly [24, 25]. Although the aforementioned mutual coherence provides a sufficiency measure to ensure sparse support recovery, it cannot measure the incoherence of the reference matrix exactly when most of the coherence values are small. Thus, a new design criterion should be proposed to optimize the reference matrix.

In matrix analysis, the condition number of a matrix measures the sensitivity of the solution of a linear system to errors in the data [26]. It gives an indication of the accuracy of the results from matrix inversion and the linear equation solution. The condition number near 1 indicates a well-conditioned matrix, which means a small modeling error induces a small imaging error. On the contrary, an illconditioned matrix will make the solution of the imaging equation sensitive to the modeling error. Furthermore, we derive the analytical expression for the upper bound of relative imaging error (RIE) in the present of modeling error and conclude that the RIE highly depends on both the modeling error and the condition number of reference matrix. As the condition number of reference matrix is closely related to $\mathrm{FH}$ code, minimizing the condition number is utilized as the design criterion to optimize the FH code. Accordingly, we derive the Gram matrix of reference matrix and optimally design the $\mathrm{FH}$ code using the quantum simulated annealing (QSA) algorithm [13].
Results of numerical simulations show our proposed $\mathrm{FH}$ code design method optimizes the reference matrix and improves the imaging performance by reducing the condition number.

The rest of the paper is organized as follows. Section 2 presents the FH-RCI model with modeling error. In Section 3, the sensitivity of RCI to modeling error is analyzed by both theoretical derivations and numerical simulations. Then the Gram matrix $\mathbf{S}^{H} \mathbf{S}$ is derived, and the $\mathrm{FH}$ code optimization method is proposed in Section 4. In Section 5, the proposed optimization method is verified by numerical simulations. Finally, Section 6 concludes the paper.

Notations used in this paper are as follows. We use boldface lowercase letters for vectors and boldface uppercase letters for matrices. $(\bullet)^{H},(\bullet)^{-1}$, and $(\bullet)^{\dagger}$ denote the conjugate transpose, inverse, and pseudo-inverse of a vector or matrix, respectively. $\|\bullet\|$ denotes the norm of a vector. Finally, $\langle\bullet\rangle$ denotes the expectation of a variable.

\section{Signal Model}

Originated from the classical optical coincidence imaging, RCI is a superresolution method within the beam based on the innovative idea of random radiation and wavefront random modulation. The essence of RCI is to produce the random-modulated wavefront by transmitting specific modulated waveforms, and that would increase the variety of temporal-spatial stochastic radiation field. Then the target information within the beam can be obtained by the coincidence processing between the target scattering echo and the stochastic radiation field [1]. Thus RCI can achieve higher resolution than the conventional radars whose resolution is limited by the Rayleigh principle.

Transmitting independent stochastic waveforms by radar array is a realizable way to generate the temporal-spatial stochastic radiation field in the imaging plane [1], as illustrated in Figure 1. In this paper, a RCI system with $M$ transmitters and one receiver is considered. The $m$ th transmitter emits a constant modulus FH waveform $S t_{m}(t)[3,4]$ shown in Figure 2. The waveform is comprised by $L$ pulses

$$
S t_{m}(t)=\sum_{l=0}^{L-1} \phi_{m}(t-l T),
$$

where $T$ denotes pulse repetition interval. $\phi_{m}(t)=$ $\sum_{q=0}^{Q-1} e^{j 2 \pi\left(f_{c}+c_{m, q} \Delta f\right) t} u(t-q \Delta t)$, where $f_{c}$ denotes the carrier frequency and

$$
u(t) \triangleq \begin{cases}1 & 0<t<\Delta t \\ 0 & \text { otherwise }\end{cases}
$$

where $\Delta t$ and $\Delta f$ denote $\mathrm{FH}$ duration and $\mathrm{FH}$ interval, respectively. $c_{m, q} \in\{0, \ldots, G-1\}$ is the $\mathrm{FH}$ code which specifies the transmitted frequency during each hopping interval, where $G$ is a positive integer. $c_{m, q}$ can be arranged into a $M \times Q$ dimensional code matrix $\mathbf{C}$ specifying the transmitted frequencies. $Q$ is the length of the code, that is, 


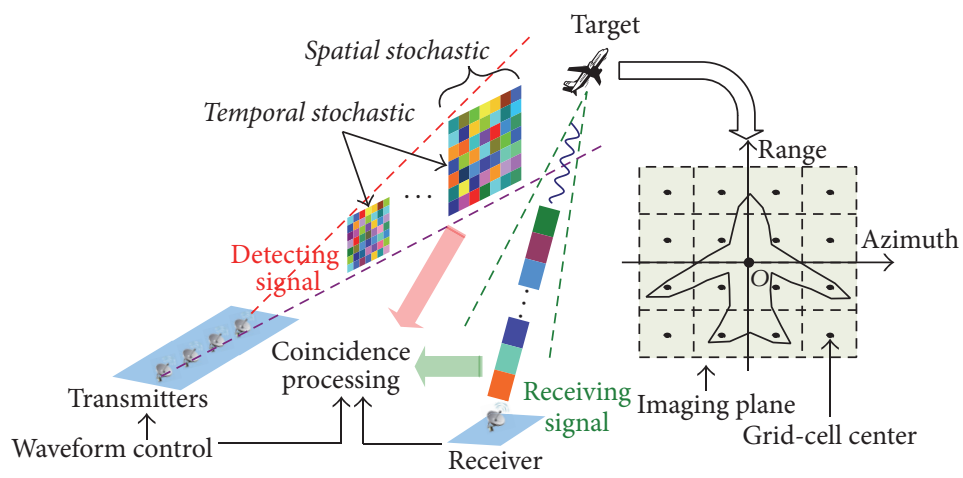

FIGURE 1: Geometry of radar coincidence imaging.

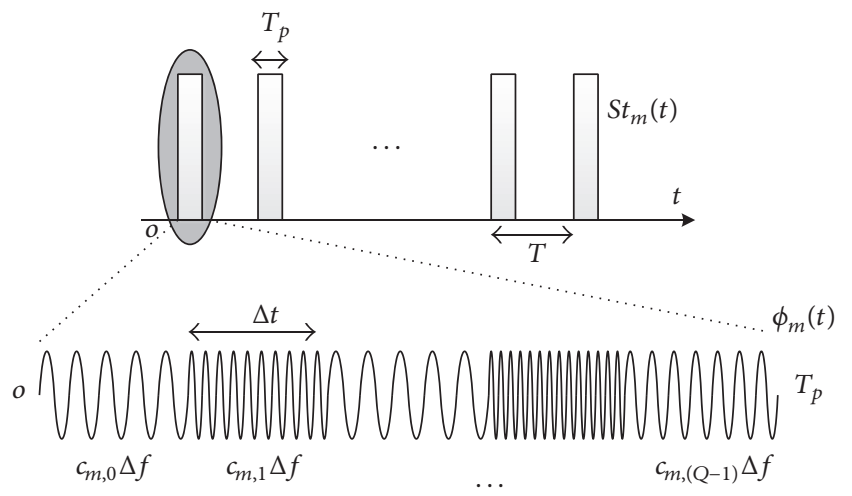

FIGURE 2: Frequency-hopping waveforms.

the total number of hopping intervals. Thus the duration of each pulse is $T_{p}=Q \Delta t$.

For FH waveform, the synthetic bandwidth of the pulses is approximately [3]

$$
B \approx(G-1) \Delta f+\frac{1}{\Delta t} .
$$

The target scene is considered to be a $2 \mathrm{D}$ range-azimuth space. Before coincidence processing, the continuous imaging plane needs to be discretized to generate $K$ grid-cells (i.e., imaging cells), and the scatterers are assumed to be located at these prediscretized grid-cell centers, that is, $\mathbf{r}_{k}$. The scatterers possess nonzero complex scattering coefficient $\beta_{k}$, and $\beta_{k}=$ 0 when there is no scatterer at grid-cell center. Denote by $\Omega$ the index set of scatterer locations; that is, $\Omega=\{k \mid$ $\left.\beta_{k} \neq 0, k \in\{1,2, \ldots, K\}\right\}$. The receiving signal is a linear combination of all the scatterers reflected waveforms from all the transmitters.

$$
y(t)=\sum_{k \in \Omega} \sum_{m=1}^{M} \beta_{k} S t_{m}\left(t-\tau_{m}^{k}\right),
$$

where $\tau_{m}^{k}=\left(\left\|\mathbf{r}_{k}-\mathbf{R}_{m}\right\|+\left\|\mathbf{r}_{k}-\mathbf{R}_{0}\right\|\right) / c$ is the propagation delay corresponding to the $m$ th transmitter and the receiver with respect to the $k$ th scatterer. $\mathbf{R}_{0}$ and $\mathbf{R}_{m}$ are the position vectors of the receiver and the $m$ th transmitter, respectively. $c$ is the speed of wave propagation.

For the sake of simplicity, the RCI formula needs a detecting signal [1], which can be simply structured as

$$
S\left(t, \mathbf{r}_{k}\right)=\sum_{m=1}^{M} S t_{m}\left(t-\tau_{m}^{k}\right) .
$$

The detecting signal $S\left(t, \mathbf{r}_{k}\right)$ can be interpreted as the signal reflected by the $k$ th grid-cell center. Then, the echo can be expressed as the superposition of the detecting signals

$$
y(t)=\sum_{k \in \Omega} \beta_{k} S\left(t, \mathbf{r}_{k}\right)
$$

After sampling the echo, the imaging equation can be given as follows:

$$
\begin{aligned}
\mathbf{y}= & \mathbf{S} \boldsymbol{\beta}, \\
{\left[\begin{array}{c}
y\left(t_{1}\right) \\
y\left(t_{2}\right) \\
\vdots \\
y\left(t_{N}\right)
\end{array}\right]=} & {\left[\begin{array}{cccc}
S\left(t_{1}, \mathbf{r}_{1}\right) & S\left(t_{1}, \mathbf{r}_{2}\right) & \cdots & S\left(t_{1}, \mathbf{r}_{K}\right) \\
S\left(t_{2}, \mathbf{r}_{1}\right) & S\left(t_{2}, \mathbf{r}_{2}\right) & \cdots & S\left(t_{2}, \mathbf{r}_{K}\right) \\
\vdots & \vdots & \cdots & \vdots \\
s\left(t_{N}, \mathbf{r}_{1}\right) & S\left(t_{N}, \mathbf{r}_{2}\right) & \cdots & S\left(t_{N}, \mathbf{r}_{K}\right)
\end{array}\right] } \\
& \cdot\left[\begin{array}{c}
\beta_{1} \\
\beta_{2} \\
\vdots \\
\beta_{K}
\end{array}\right],
\end{aligned}
$$

where $\mathbf{y}$ and $\beta$ are the receiving signal and scattering coefficient vector, respectively. $t_{N}$ denotes the sampling time. $\mathbf{S}$ is the reference matrix comprised by the detecting signals at different grid-cell centers. The columns and the rows of $\mathbf{S}$ basically represent the detecting signals at different positions and different instants, respectively, which could present the temporal-spatial stochastic characteristics of radiation field. 
Consequently, target reconstruction reduces to a linear inverse problem which recovers $\boldsymbol{\beta}$ from the measurement vector $\mathbf{y}$ and reference matrix $\mathbf{S}$. First of all, the reference matrix should be calculated accurately, and the imaging performance depends on the incoherence property of $\mathbf{S}$. However, the reference matrix obtained from practical applications is inevitably perturbed by the modeling error which cannot be known accurately in practice. Consider the modeling error, the true reference matrix is expressed as $\widehat{\mathbf{S}}=$ $\mathbf{S}+\Delta \mathbf{S}$, where $\mathbf{S}$ is the known nominal reference matrix and $\Delta \mathbf{S}$ represents the unknown matrix perturbation. Then the true coincidence imaging equation can be decomposed as follows:

$$
\mathbf{y}=\widehat{\mathrm{S}} \cdot \boldsymbol{\beta}=\mathrm{S} \boldsymbol{\beta}+\Delta \mathrm{S} \boldsymbol{\beta}
$$

Thus, the multiplicative term $\Delta \mathbf{S} \boldsymbol{\beta}$ is introduced into the perturbed RCI, which is difficult to analyze since it is correlated with the signal of interest [27].

\section{Sensitivity Analysis of RCI to Modeling Error}

3.1. Target Reconstruction. There are several approaches to retrieve $\boldsymbol{\beta}$ from $\mathbf{y}$. The simplest one is named matchedfiltering and consists in performing the following matrix multiplication:

$$
\overline{\boldsymbol{\beta}}=\mathbf{S}^{H} \bullet \mathbf{y} .
$$

This approach works decently by maximizing the postprocessing signal-to-noise ratio (SNR) in radar echoes. Thus, matched-filtering is less sensitive to measurement noise. However, only the correlation between $\mathbf{y}$ and $\mathbf{S}$ cannot generate a high-resolution image for RCI, because the practical microwave waveform cannot generate complete temporalspatial stochastic radiation field due to the limited bandwidth and array aperture. Fortunately, the linear inverse model in (7) can be also solved using optimization algorithms, while $\|\mathbf{y}-\mathbf{S} \cdot \boldsymbol{\beta}\|_{2}^{2}$ is used as the objective function. An intuitionistic approach is to employ the least square (LS) estimation, which is expressed as

$$
\widehat{\boldsymbol{\beta}}=\underset{\boldsymbol{\beta}}{\arg \min }\|\mathbf{y}-\mathbf{S} \cdot \boldsymbol{\beta}\|_{2}^{2}
$$

The solution of LS estimator is $\widehat{\beta}=\mathbf{S}^{\dagger} \mathbf{y}$, where $\mathbf{S}^{\dagger}=$ $\left(\mathbf{S}^{H} \mathbf{S}\right)^{-1} \mathbf{S}^{H}$ is the pseudo-inverse of $\mathbf{S}$. Based on the LS principle, the target image can be reconstructed. It is worthy noticing that the LS estimator is optimal when the noise is Gaussian. By simply calculating the pseudo-inverse of $\mathbf{S}$, the LS method is easy to implement with potential in highresolution imaging. However, the LS method is still constrained by the temporal-spatial stochastic characteristics of radiation field, and it has a low tolerance to the modeling error and noise especially when the reference matrix is illconditioned. Once the imaging equation (7) is perturbed by the modeling error, the optimization process would be obviously disturbed, and the error will be directly presented in the solution. Herein, the sensitivity of the LS method to the modeling error would make the target reconstruction unstable.

By adding the regularization terms, the target reconstruction would become more stable, and the Tikhonov regularization algorithm emerges $[28,29]$. When the target image is sparse, sparse reconstruction algorithm can be used to achieve superresolution, denoising, and feature extraction, by exploiting the sparse prior [30].

3.2. Sensitivity Analysis. To explore the characteristics of the reference matrix $\mathbf{S}$, singular value decomposition (SVD) is employed as

$$
\mathbf{S}=\mathbf{U} \mathbf{\Sigma} \mathbf{V}^{H}
$$

where $\mathbf{U}$ and $\mathbf{V}$ are unitary singular vector matrices and $\boldsymbol{\Sigma}=$ $\operatorname{diag}\left(\sigma_{1}, \sigma_{2}, \ldots, \sigma_{r}, 0, \ldots\right)$ consists of the nonnegative, nonincreasingly ordered singular values on its diagonal entries. $r$ is the number of nonzero singular values. Then the scattering coefficient vector can be estimated as

$$
\begin{aligned}
\widehat{\boldsymbol{\beta}} & =\mathbf{S}^{\dagger}(\mathbf{S} \boldsymbol{\beta}+\Delta \mathbf{S} \boldsymbol{\beta}) \\
& =\left(\sum_{k=1}^{r} \mathbf{u}_{k} \sigma_{k} \mathbf{v}_{k}^{H}\right)^{\dagger}\left(\left(\sum_{k=1}^{r} \mathbf{u}_{k} \sigma_{k} \mathbf{v}_{k}^{H}\right) \boldsymbol{\beta}+\Delta \mathbf{S} \boldsymbol{\beta}\right) \\
& =\sum_{k=1}^{r} \mathbf{v}_{k} \mathbf{v}_{k}^{H} \boldsymbol{\beta}+\sum_{k=1}^{r} \mathbf{v}_{k} \sigma_{k}^{-1} \mathbf{u}_{k}^{H} \Delta \mathbf{S} \boldsymbol{\beta} \\
& =\left(\mathbf{I}-\sum_{k=r+1}^{K} \mathbf{v}_{k} \mathbf{v}_{k}^{H}\right) \boldsymbol{\beta}+\sum_{k=1}^{r} \mathbf{v}_{k} \sigma_{k}^{-1} \mathbf{u}_{k}^{H} \Delta \mathbf{S} \boldsymbol{\beta} .
\end{aligned}
$$

The first term $\left(\mathbf{I}-\sum_{k=r+1}^{K} \mathbf{v}_{k} \mathbf{v}_{k}^{H}\right) \boldsymbol{\beta}$ in (12) represents the approximate of $\boldsymbol{\beta}$, indicating that the LS estimation of underdetermined problem results in the approximation of actual value. The second term, $\sum_{k=1}^{r} \mathbf{v}_{k} \sigma_{k}^{-1} \mathbf{u}_{k}^{H} \Delta \mathbf{S} \boldsymbol{\beta}$, is the perturbation resulting from the modeling error. Due to the limited bandwidth of transmissions and array aperture, the temporal-spatial incoherence of radiation field is not perfect; therefore some singular values tend to be quite small, which means $s$ small modeling error $\Delta \mathbf{S}$ would induce a terrible perturbation of $\boldsymbol{\beta}$. Accordingly, the singular value should be increased to depress the effect of modeling error $\Delta \mathbf{S}$.

In matrix analysis, the condition number is commonly used as a measure of numerical stability for the linear equation; that is, $\mathbf{y}=\mathbf{S} \bullet \boldsymbol{\beta}$. Intuitively speaking, the condition number depicts the perturbation degree that $\beta$ is scaled by the perturbation $\Delta \mathbf{S}$, a small condition number will weaken the impact of the perturbation $\Delta \mathbf{S}$, and a large one will enhance this impact. The condition number of $\mathbf{S}$ is defined as the ratio of the maximum to minimum singular values of $\mathbf{S}$ [31]

$$
\operatorname{cond}(\mathbf{S})=\frac{\sigma_{\max }}{\sigma_{\min }},
$$


where $\sigma_{\max }$ and $\sigma_{\min }$ denote the maximum and minimum singular values, respectively.

Next, the condition number is employed to define the upper bound of imaging error in the presence of modeling error.

In practice RCI applications, the true reference matrix $\widehat{\mathbf{S}}$ cannot be employed in practice, as the prior knowledge of the imaging model is hardly to be known accurately. Then the estimated imaging equation actually employed in practice is

$$
\mathrm{y}=\mathrm{S} \cdot \overline{\boldsymbol{\beta}},
$$

where $\widehat{\beta}$ is the estimated scattering coefficient vector. Denote by $\boldsymbol{\varepsilon}=\boldsymbol{\beta}-\widehat{\boldsymbol{\beta}}$ the imaging error, where $\boldsymbol{\beta}$ is true scattering coefficient vector. Deriving from (8) and (14), we have (S + $\Delta \mathbf{S}) \bullet \boldsymbol{\beta}=\mathbf{S} \bullet(\boldsymbol{\beta}-\boldsymbol{\varepsilon})$. Then the imaging error can be expressed as

$$
\varepsilon=-\mathbf{S}^{\dagger} \cdot \Delta \mathbf{S} \cdot \boldsymbol{\beta}
$$

If the number of measurements is equal to that of gridcells, that is, $N=K, \mathbf{S}$ is a square matrix, and (15) reduces to $\boldsymbol{\varepsilon}=-\mathbf{S}^{-1} \cdot \Delta \boldsymbol{S} \cdot \boldsymbol{\beta}$. Taking norms according to the matrix norm consistent, we have

$$
\|\varepsilon\| \leq\left\|S^{-1}\right\| \cdot\|\Delta S\| \cdot\|\beta\|=\left\|S^{-1}\right\|\|S\| \cdot \frac{\|\Delta S\|}{\|S\|} \bullet\|\beta\| .
$$

The term $\left\|\mathbf{S}^{-1}\right\|\|\mathbf{S}\|$ in (16) is also defined as the condition number of $\mathbf{S}$, that is, $\operatorname{cond}(\mathbf{S})=\left\|\mathbf{S}^{-1}\right\|\|\mathbf{S}\|[31]$. So the relative imaging error (RIE) can be obtained as

$$
\frac{\|\varepsilon\|}{\|\beta\|} \leq \operatorname{cond}(\mathbf{S}) \cdot \frac{\|\Delta \mathbf{S}\|}{\|\mathbf{S}\|} .
$$

When $N>K$, the number of equations is more than that of unknown parameters, and (7) becomes an overdetermined problem. The pseudo-inverse of $\mathbf{S}$ in (15) is then defined as $\mathbf{S}^{\dagger}=\left(\mathbf{S}^{H} \mathbf{S}\right)^{-1} \mathbf{S}^{H}$. Thus $\boldsymbol{\varepsilon}$ becomes

$$
\begin{aligned}
\|\boldsymbol{\varepsilon}\| & \leq\left\|\left(\mathbf{S}^{H} \mathbf{S}\right)^{-1}\right\| \cdot\left\|\mathbf{S}^{H} \Delta \mathbf{S}\right\| \cdot\|\boldsymbol{\beta}\| \\
& =\left\|\left(\mathbf{S}^{H} \mathbf{S}\right)^{-1}\right\|\left\|\mathbf{S}^{H} \mathbf{S}\right\| \frac{\left\|\mathbf{S}^{H} \Delta \mathbf{S}\right\|}{\left\|\mathbf{S}^{H} \mathbf{S}\right\|} \bullet\|\boldsymbol{\beta}\| \\
& =\operatorname{cond}\left(\mathbf{S}^{H} \mathbf{S}\right) \frac{\left\|\mathbf{S}^{H} \Delta \mathbf{S}\right\|}{\left\|\mathbf{S}^{H} \mathbf{S}\right\|} \cdot\|\boldsymbol{\beta}\| .
\end{aligned}
$$

Because $\left\|\mathbf{S}^{H} \mathbf{S}\right\|=\|\mathbf{S}\|^{2}$, the RIE can be obtained as

$$
\frac{\|\varepsilon\|}{\|\beta\|} \leq \operatorname{cond}\left(S^{H} \mathbf{S}\right) \frac{\|\Delta S\|}{\|S\|}
$$

Note that, for positive semidefinite Hermitian matrix $\mathbf{S}^{H} \mathbf{S}$, its condition number doubles cond $(\mathbf{S})$; that is,

$$
\operatorname{cond}\left(\mathbf{S}^{H} \mathbf{S}\right)=(\operatorname{cond}(\mathbf{S}))^{2} \text {. }
$$

The right side of (17) and (19) defines the upper bound of RIE, which could be used as an indication to analyze the influence factors. Obviously, two key factors determine the upper bound of the RIE, that is, the condition number and modeling error.

(1) Modeling Error. The norm of modeling error $\|\Delta \mathbf{S}\|$ is an important factor degrading the imaging performance. Certainly, a smaller modeling error $\Delta \mathbf{S}$ means a minor difference between $\widehat{\mathbf{S}}$ and $\mathbf{S}$ and will generate a smaller RIE when the condition number of reference matrix is fixed. Unfortunately, even a small system error, for example, gainphase error and array position error, will generate a large modeling error $[2,14,23]$. To minimize the modeling error, we should decrease the RCI system error or obtain the system parameters accurately. Otherwise, the target reconstruction algorithm should be implemented to compensate the modeling error $[2,14-16,22,23]$.

(2) Condition Number. As shown in (17) and (19), cond(S) or cond $\left(\mathbf{S}^{H} \mathbf{S}\right)$ can be viewed as the amplification factor of $\|\Delta \mathbf{S}\| /\|\mathbf{S}\|$. A small condition number will weaken the impact of $\Delta \mathbf{S}$, and contrarily, a large one will enhance this impact. Besides, as presented in (20), the condition number of an overdetermined equation is doubled; thus the condition number shows more significant influence on the RIE for an overdetermined system.

In RCI formulation, the condition number cond(S) measures the incoherence of the reference matrix $\mathbf{S}$. The bigger the condition number, the weaker the incoherence degree the row/column vectors of the matrix present. Thus, the condition number cond(S) essentially depends on the temporal-spatial stochastic characteristics of radiation field. The radiation field of higher temporal-spatial stochastic degree leads to a more incoherent matrix $\mathbf{S}$, resulting in a smaller cond(S) to decrease the RIE.

3.3. Numerical Simulations. The following example concerns how the condition number of $\mathbf{S}$ and the modeling error $\Delta \mathbf{S}$ affect the imaging performance. The imaging experiment will be performed in the scenarios where the modeling error and condition number are different. For the sake of discussion, we quantify the modeling error with the relative modeling error $(\mathrm{RME}): \mathrm{RME}=20 \log _{10}(\|\Delta \mathbf{S}\| /\|\mathbf{S}\|)$, which represents the relative perturbation error between $\|\Delta \mathbf{S}\|$ and $\|\mathbf{S}\|$. The imaging quality is indicated by the RIE, which is expressed as $\mathrm{RIE}=20 \log _{10}(\|\boldsymbol{\varepsilon}\| /\|\boldsymbol{\beta}\|)$.

In the simulations, the RCI system works at the Xband with carrier frequency of $10 \mathrm{GHz}$. The transmitters are configured as a uniform linear array (ULA) with 16 transmitters. A range-azimuth imaging plane is discretized to $40 \times 40$ grid-cells. The transmitters emit independent $\mathrm{FH}$ waveforms comprised by 2500 hopping frequencies with the minimum $\mathrm{FH}$ interval $1 \mathrm{MHz}$ and $\mathrm{FH}$ duration $10 \mathrm{~ns}$.

Although we focus on the design of $\mathrm{FH}$ code in order to minimize the condition number, it would be meaningful to show numerically the condition number behavior in terms of signal bandwidth, grid-cell size, and array aperture. The result is presented in Figure 3, where signal bandwidth $B \in\{0.25 \mathrm{GHz}, 0.5 \mathrm{GHz}, 1 \mathrm{GHz}\}$, grid-cell size changes from 


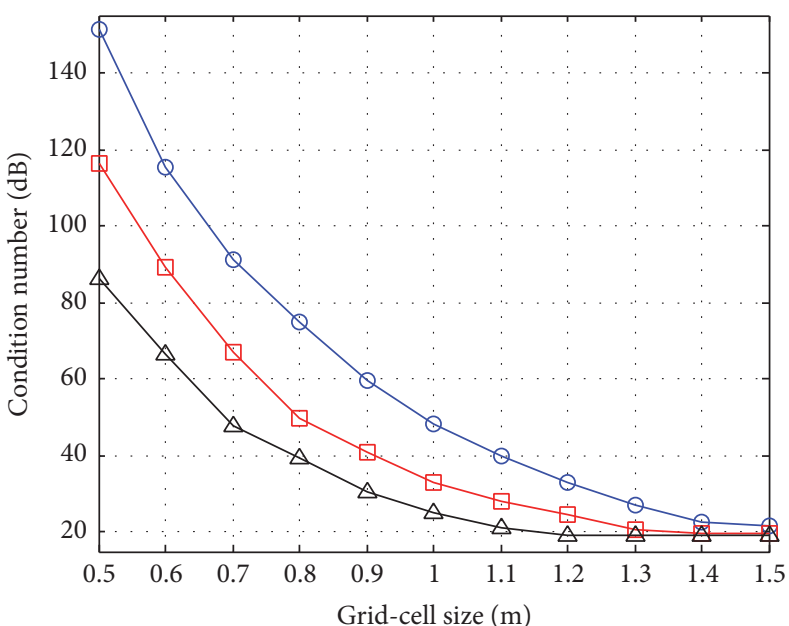

$$
\begin{aligned}
-B & =0.25 \mathrm{GHz} \\
\square & =0.5 \mathrm{GHz} \\
\triangle B & =1 \mathrm{GHz}
\end{aligned}
$$

(a)

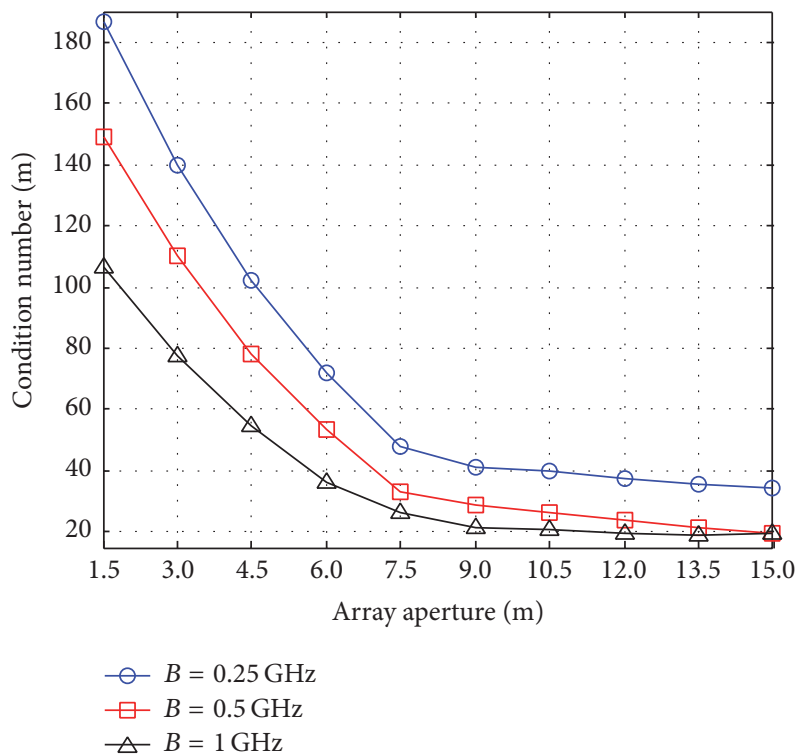

(b)

Figure 3: Condition number in terms of signal bandwidth, grid-cell size, and array aperture. (a) Condition number versus grid-cell size for different signal bandwidths. (b) Condition number versus array aperture for different signal bandwidths.

$0.5 \mathrm{~m} \times 0.5 \mathrm{~m}$ to $1.5 \mathrm{~m} \times 1.5 \mathrm{~m}$, and the array aperture varies from $1.5 \mathrm{~m}$ to $15 \mathrm{~m}$ by changing the interelement spacing. As shown in Figure 3, increasing the bandwidth of $\mathrm{FH}$ waveform makes the reference matrix more incoherent and accordingly the condition number become small. Similarly, the incoherence between the detecting signals at neighboring grid-cell centers is enhanced when the grid-cell size and array aperture increase, which induces a smaller condition number of reference matrix.

The first example is to investigate the influence of condition number on the imaging quality. The bandwidth of FH waveforms is changed to generate different incoherence degrees of the reference matrix; thus the condition number varies. The RCI results are depicted in Figure 4, where the modeling error $\Delta \mathbf{S}$ is a Gaussian process with RME $=-20 \mathrm{~dB}$. Figure 4(a) is the target with 77 scatterers; Figures 4(b)4(d) are the RCI results of LS method for different condition numbers. Obviously, the performance of LS method degrades as the condition number of $\mathbf{S}$ becomes larger, because the imaging equation (7) tends to be ill-posed and the solution of LS estimation becomes unstable which means even a small modeling error would induce a large imaging error.

To investigate the distributions of singular values for different condition numbers, we plot the histogram of the normalized singular values, as depicted in Figure 5. As seen from the figure, increasing the bandwidth would make the singular centralized; thus the condition number $\sigma_{\max } / \sigma_{\min }$ becomes small. Besides, a large signal bandwidth results in the increasing number of large singular values, which means the incoherence of the detecting signals is improved.
Next, we simulate the influence of modeling error $\Delta \mathbf{S}$ on the imaging quality. The condition number of $\mathbf{S}$ is fixed at $33.11 \mathrm{~dB}$, while the RME varies from $-10 \mathrm{~dB}$ to $-40 \mathrm{~dB}$. Figure 6 depicts the imaging results. We can conclude that RCI has a low tolerance to modeling error, and small modeling error exhibits significant performance improvement with high-resolution enhancement. Contrarily, increasing the modeling error would obtain blurred reconstructed images with many spurious scatterers.

\section{Waveform Optimization for RCI}

Based on the discussion in Section 3, we conclude that the imaging performance depends on the incoherence property of the reference matrix, that is, the temporal-spatial stochastic characteristics of the detecting signals. In this section, the FH code is designed by optimizing the reference matrix $\mathbf{S}$.

4.1. Optimization Criterion. As discussed in Section 3, the condition number could measure the numerical stability of the linear equation (7). Small condition number means that the reference matrix is well-conditioned and is often desired with respect to numerical robustness regarding matrix inverse and decomposition. Furthermore, when there exists modeling error, the performance of RCI with large condition number is degraded significantly. Thus, decreasing the condition number of $\mathbf{S}$ would improve the robustness of RCI. Unfortunately, the matrix incoherence or cond(S) depends on the temporal-spatial stochastic characteristics of detecting signals, which is determined by many factors, for instance, the FH code, signal bandwidth, array aperture size, and grid-cell 


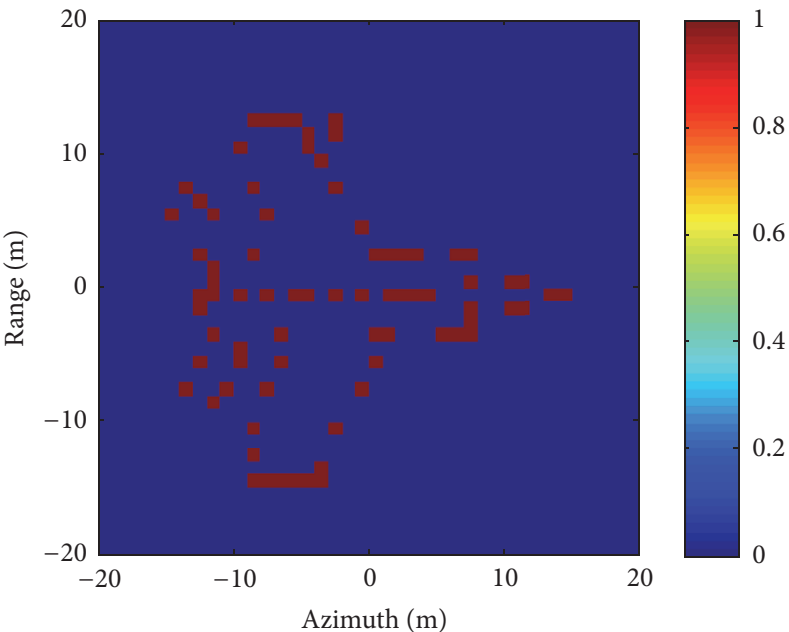

(a)

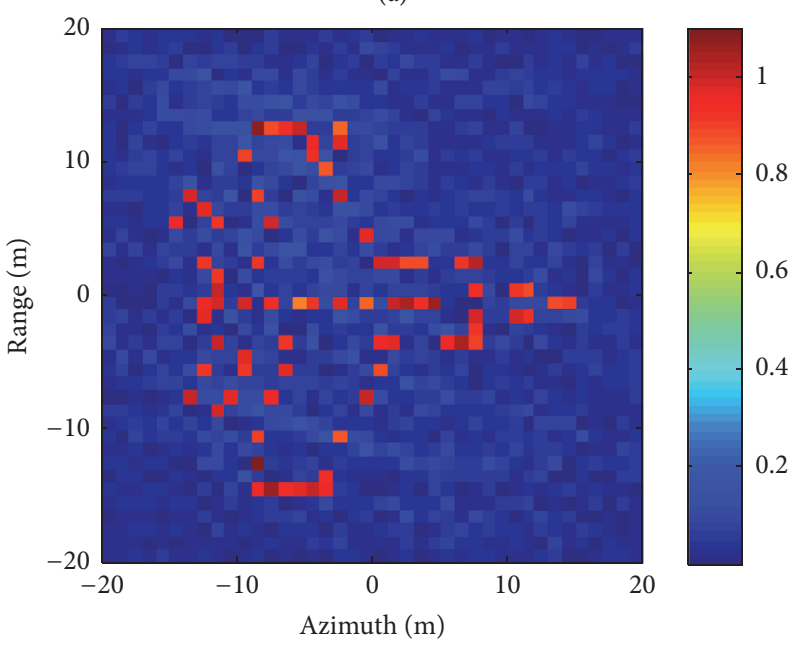

(c)

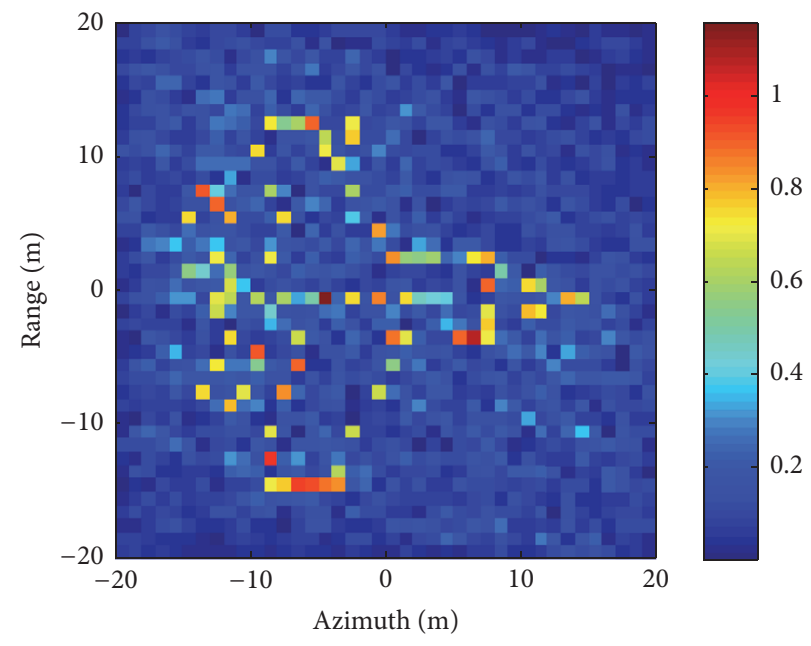

(b)

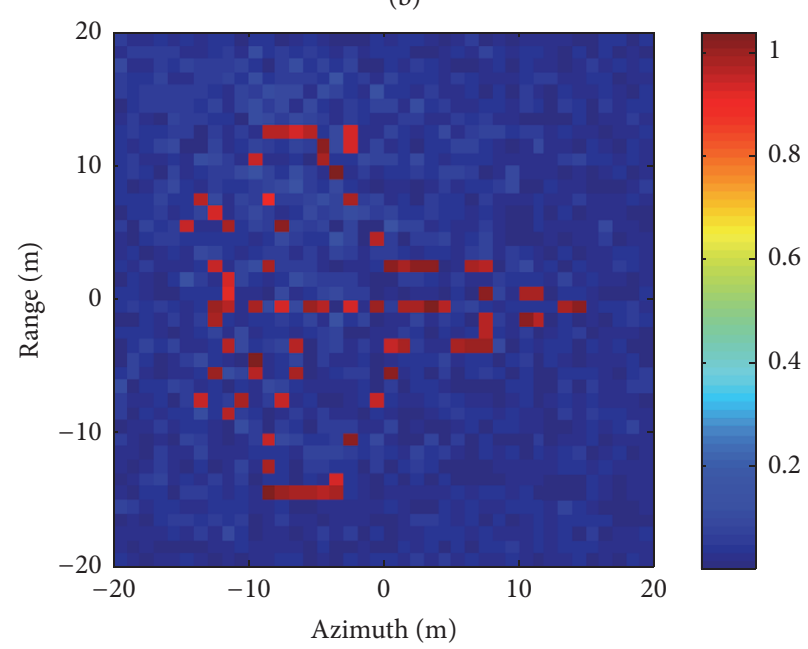

(d)

FIGURE 4: RCI results for different condition numbers when RME $=-20 \mathrm{~dB}$. (a) Target model; (b) cond(S) $=54.49 \mathrm{~dB}, \mathrm{RIE}=-3.03 \mathrm{~dB}$; (c) $\operatorname{cond}(\mathbf{S})=33.29 \mathrm{~dB}, \mathrm{RIE}=-11.02 \mathrm{~dB} ;(\mathrm{d}) \operatorname{cond}(\mathbf{S})=28.22 \mathrm{~dB}, \mathrm{RIE}=-14.05 \mathrm{~dB}$.

size. Herein, the detecting signals are incompletely temporalspatial stochastic in practical microwave imaging. Thus the real-world reference matrix is often ill-conditioned or even rank-deficient, resulting in serious numerical difficulties and performance losses.

However, it is worthy noticing that each column of $\mathbf{S}$ corresponds to all transmitters and all FH codes; the RCI system with different $\mathrm{FH}$ codes would generate the radiation field with diverse incoherence degrees. Consequently, the $\mathrm{FH}$ codes can be designed to optimize the reference matrix with large singular values downwards and small ones upwards; then the condition number decreases. Thus the minimization of the condition number of $\mathbf{S}^{H} \mathbf{S}$ is considered as the design criterion, and the object function can be written as $f(\mathbf{C})=\operatorname{cond}\left(\mathbf{S}^{H} \mathbf{S}\right)$. The corresponding optimization problem is expressed as

$$
\begin{aligned}
\mathbf{C}_{\text {opt }}=\underset{\mathbf{C}}{\arg \min } & f(\mathbf{C}) \\
\text { s.t. } & \mathbf{C} \in\{0,1, \ldots, G-1\} .
\end{aligned}
$$

The element of Gram matrix $\mathbf{S}^{H} \mathbf{S}$ is the mutual coherence of detecting signals with respect to different grid-cells. Next, the relation of Gram matrix $\mathbf{S}^{H} \mathbf{S}$ and $\mathrm{FH}$ code $\mathbf{C}$ will be derived to show how $\mathrm{FH}$ code affects the mutual coherent.

4.2. Derivation of Coherence Measure. To calculate the condition number of $\mathbf{S}^{H} \mathbf{S}$, we should first derive the correlation between the different columns of the reference matrix $\mathbf{S}$, that is, $\mathbf{S}_{k}^{H} \cdot \mathbf{S}_{k^{\prime}} \cdot \mathbf{S}_{k}^{H} \cdot \mathbf{S}_{k^{\prime}}$ is the summation over time samples, which is difficult to compute its analytical expression. Alternatively, we make the integral (22), which approximates $\mathbf{S}_{k}^{H} \cdot \mathbf{S}_{k^{\prime}}$ well especially in case of high sampling rate.

$$
\begin{aligned}
& \left\langle\mathbf{S}_{k}, \mathbf{S}_{k^{\prime}}\right\rangle \cong \int_{-\infty}^{\infty} S\left(t, \mathbf{r}_{k}\right) S^{*}\left(t, \mathbf{r}_{k^{\prime}}\right) d t \\
& \quad=\int_{-\infty}^{\infty}\left(\sum_{m, m^{\prime}=1}^{M} S t_{m}\left(t-\tau_{m}^{k}\right) S t_{m^{\prime}}^{*}\left(t-\tau_{m^{\prime}}^{k^{\prime}}\right)\right) d t .
\end{aligned}
$$




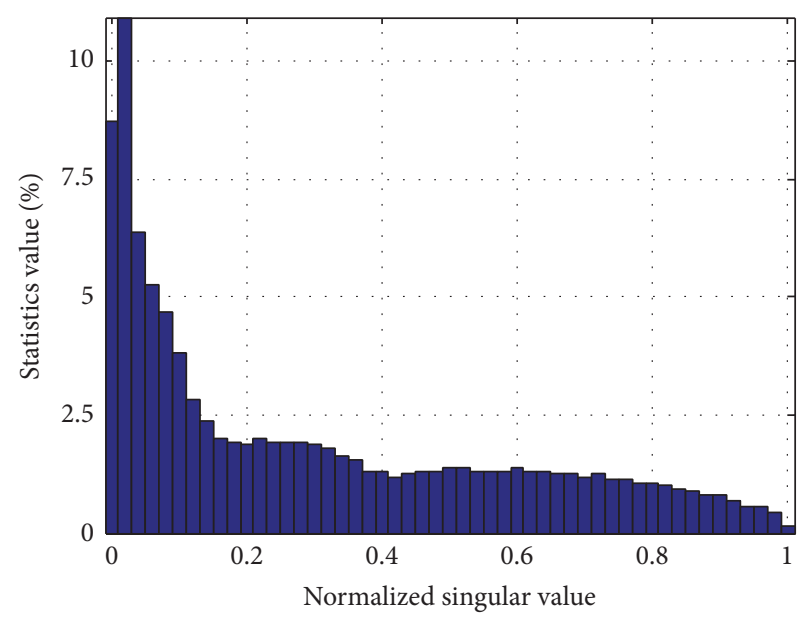

(a)

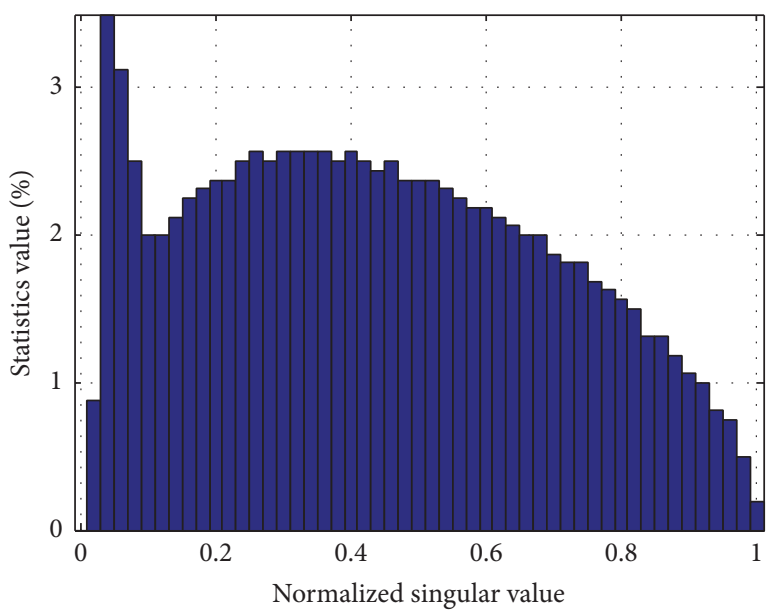

(b)

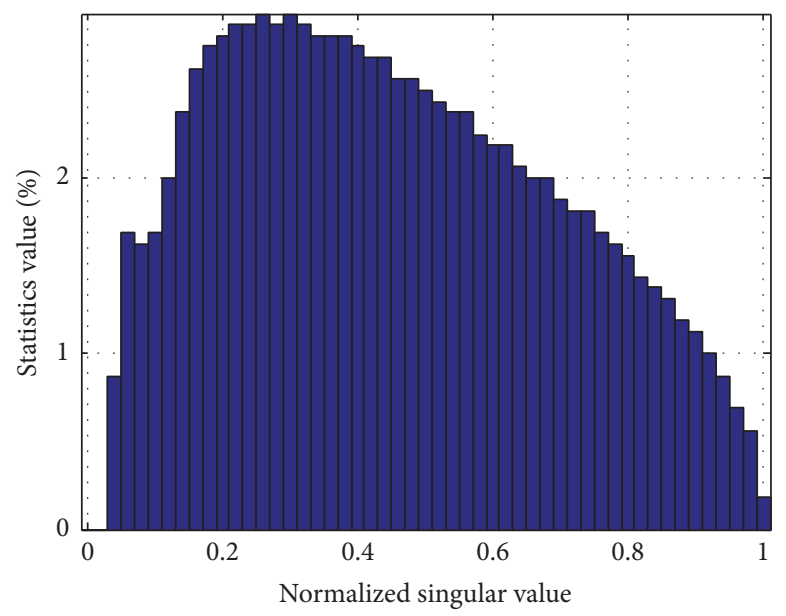

(c)

FIGURE 5: Histogram of the normalized singular values for different condition numbers. (a) cond(S) $=54.49 \mathrm{~dB}$; (b) cond(S) = $33.29 \mathrm{~dB}$; (c) $\operatorname{cond}(\mathbf{S})=28.22 \mathrm{~dB}$.

Define $\chi_{m, m^{\prime}}\left(\tau_{m}^{k}, \tau_{m^{\prime}}^{k^{\prime}}\right) \triangleq \int_{-\infty}^{\infty} S t_{m}\left(t-\tau_{m}^{k}\right) S t_{m^{\prime}}^{*}\left(t-\tau_{m^{\prime}}^{k^{\prime}}\right) d t$ For FH waveforms, $\chi_{m, m^{\prime}}\left(\tau_{m}^{k}, \tau_{m^{\prime}}^{k^{\prime}}\right)$ becomes

$$
\chi_{m, m^{\prime}}\left(\tau_{m}^{k}, \tau_{m^{\prime}}^{k^{\prime}}\right) \triangleq \sum_{l, l^{\prime}=1}^{L} \chi_{m, m^{\prime}}^{\phi}\left(l T+\tau_{m}^{k}, l^{\prime} T+\tau_{m^{\prime}}^{k^{\prime}}\right)
$$

where $\chi_{m, m^{\prime}}^{\phi}\left(\tau_{m}^{k}, \tau_{m^{\prime}}^{k^{\prime}}\right)=\int_{0}^{T_{\phi}} \phi_{m}\left(t-\tau_{m}^{k}\right) \phi_{m^{\prime}}^{*}\left(t-\tau_{m^{\prime}}^{k^{\prime}}\right) d t$ is the cross-correlation function. Assume that the maximum expected delay difference is less than the time interval between any two consecutive pulses of each transmitter waveform as they are collocated [13]. This assumption implies that no reflections occur at the second trip ranges and $\mid \tau_{m}^{k}-$ $\tau_{m^{\prime}}^{k^{\prime}} \mid<T-T_{\phi}$ due to $\chi_{m, m^{\prime}}^{\phi}\left(l T+\tau_{m}^{k}, l^{\prime} T+\tau_{m^{\prime}}^{k^{\prime}}\right)=0$, for $l \neq l^{\prime}$. Hence

$$
\chi_{m, m^{\prime}}\left(\tau_{m}^{k}, \tau_{m^{\prime}}^{k^{\prime}}\right)=\chi_{m, m^{\prime}}^{\phi}\left(\tau_{m}^{k}, \tau_{m^{\prime}}^{k^{\prime}}\right)
$$

After some derivations, the cross-correlation between two individual pulses of different waveforms $\phi_{m}(t)$ and $\phi_{m^{\prime}}(t)$ can be expressed as

$$
\begin{aligned}
& \chi_{m, m^{\prime}}^{\phi}(\tau)=\sum_{q, q^{\prime}=0}^{\mathrm{Q}-1} e^{j 2 \pi v q \Delta t} e^{-j 2 \pi\left(f_{c}+c_{m^{\prime}, q^{\prime}} \Delta f\right) \tau} \chi^{\mathrm{rect}}(\tau \\
&\left.-\left(q^{\prime}-q\right) \Delta t, v\right),
\end{aligned}
$$

where $\tau=\tau_{m}^{k}-\tau_{m^{\prime}}^{k^{\prime}}$ and $v=\left(c_{m, q}-c_{m^{\prime}, q^{\prime}}\right) \Delta f \cdot \chi^{\text {rect }}(\tau, v)$ is given by

$$
\chi^{\mathrm{rect}}(\tau, v) \triangleq \int_{0}^{\Delta t} u(t) u(t+\tau) e^{j 2 \pi v t} d t .
$$

When $\tau>0, \chi^{\text {rect }}(\tau, v)$ becomes

$$
\begin{aligned}
\chi^{\mathrm{rect}}(\tau, v) & =\int_{0}^{\Delta t-\tau} e^{j 2 \pi v t} d t \\
& =(\Delta t-\tau) \operatorname{sinc}(\pi v(\Delta t-\tau)) e^{j \pi v(\Delta t-\tau)} .
\end{aligned}
$$




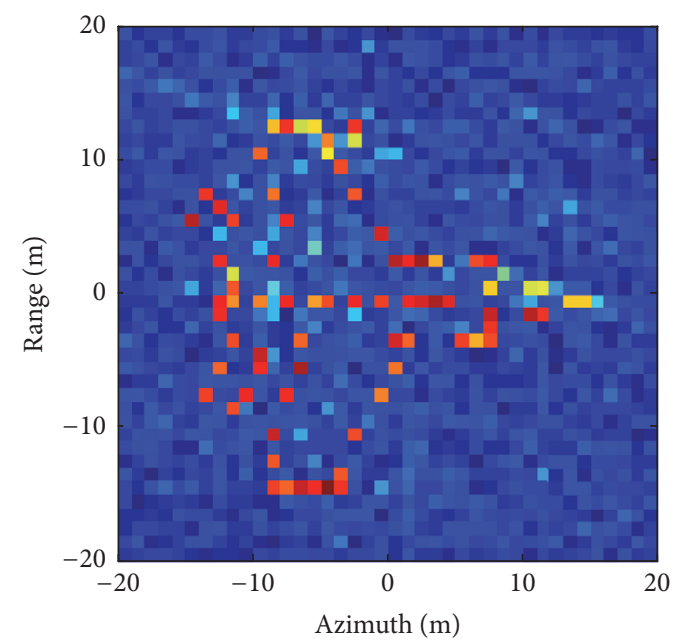

(a)

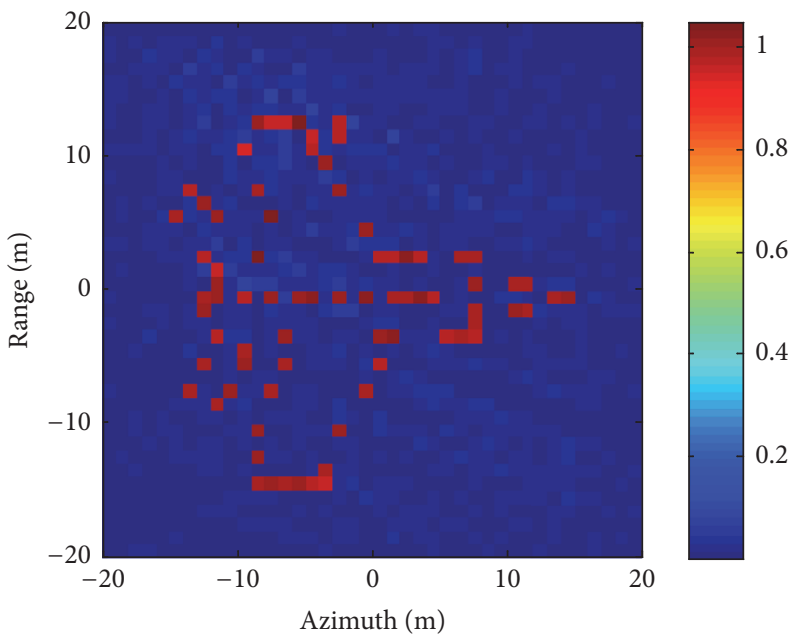

(c)
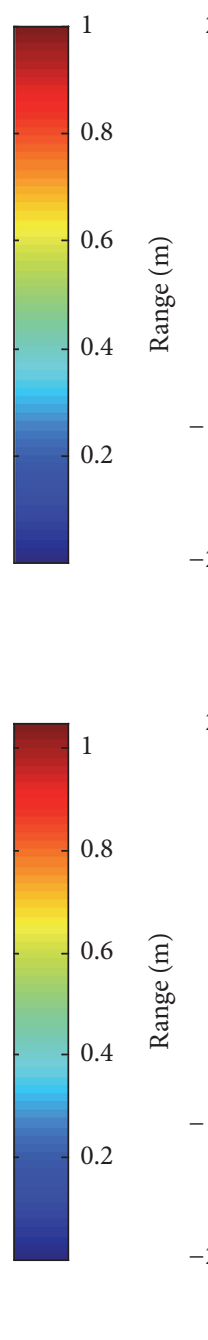

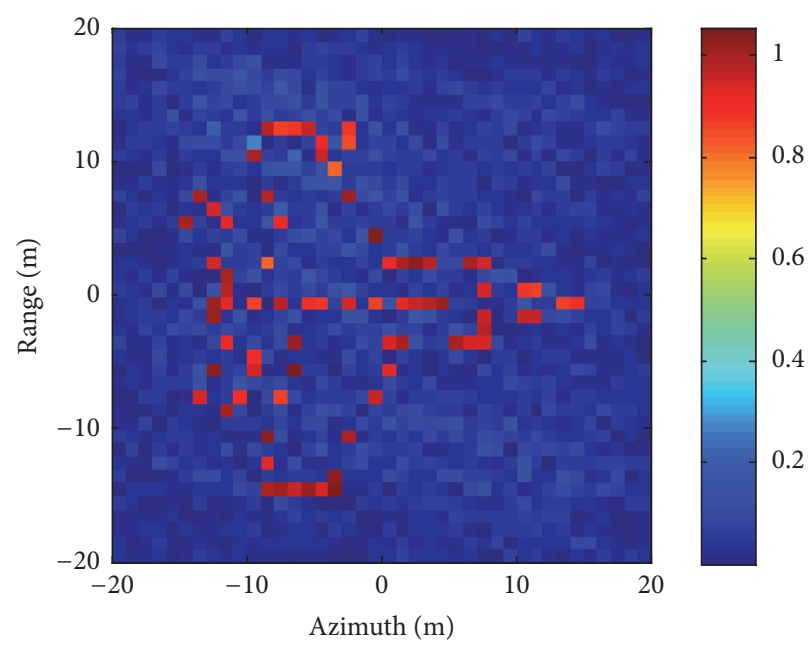

(b)

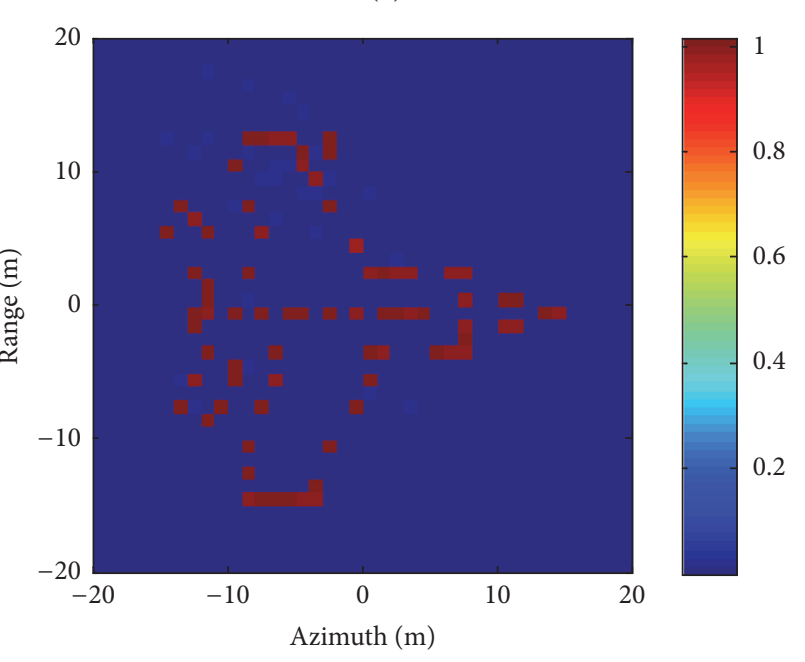

(d)

FIGURE 6: RCI results for different RMEs when $\operatorname{cond}(\mathbf{S})=33.11 \mathrm{~dB}$. (a) RME $=-10 \mathrm{~dB}, \mathrm{RIE}=-4.43 \mathrm{~dB}$; (b) RME $=-20 \mathrm{~dB}, \mathrm{RIE}=$ $-10.84 \mathrm{~dB}$; (c) RME $=-30 \mathrm{~dB}, \mathrm{RIE}=-20.08 \mathrm{~dB}$; (d) RME $=-40 \mathrm{~dB}$, RIE $=-30.12 \mathrm{~dB}$.

$$
\begin{aligned}
& \text { For } \tau<0, \chi^{\text {rect }}(\tau, v) \text { becomes } \\
& \begin{aligned}
\chi^{\text {rect }}(\tau, v) & =\int_{-\tau}^{\Delta t} e^{j 2 \pi v t} d t \\
& =(\Delta t+\tau) \operatorname{sinc}(\pi v(\Delta t+\tau)) e^{j \pi v(\Delta t-\tau)} .
\end{aligned}
\end{aligned}
$$

$$
\begin{aligned}
& \text { Combining (27) and (28), } \chi^{\text {rect }}(\tau, v) \text { is given by } \\
& \chi^{\text {rect }}(\tau, v) \\
& = \begin{cases}(\Delta t-|\tau|) \operatorname{sinc}(\pi v(\Delta t-|\tau|)) e^{j \pi v(\Delta t-\tau)} & |\tau|<\Delta t \\
0 & \text { otherwise. }\end{cases}
\end{aligned}
$$

Finally, $\left\langle\mathbf{S}_{k}, \mathbf{S}_{k^{\prime}}\right\rangle$ can be expressed as

$$
\left\langle\mathbf{S}_{k}, \mathbf{S}_{k^{\prime}}\right\rangle= \begin{cases}\sum_{m, m^{\prime}}^{M} \sum_{q, q^{\prime}=0}^{Q-1}\left(e^{j 2 \pi v q \Delta t} e^{-j 2 \pi\left(f_{c}+c_{m \prime, q^{\prime}} \Delta f\right) \tau} e^{j \pi v(\Delta t-\tau)} \times(\Delta t-|\tau|) \operatorname{sinc}(\pi v(\Delta t-|\tau|))\right) & |\tau|<\Delta t \\ 0 & \text { otherwise }\end{cases}
$$

Equation (30) implies that the correlation between the different columns of the reference matrix $\mathbf{S}$ is closely related with the FH code $\mathbf{C}$. Since $\left\langle\mathbf{S}_{k}, \mathbf{S}_{k^{\prime}}\right\rangle\left(k, k^{\prime}=1, \ldots, K\right)$ comprises the Gram matrix $\mathbf{S}^{H} \mathbf{S}$, it can be concluded that 
the choice of FH code $\mathbf{C}$ determines the singular of $\mathbf{S}^{H} \mathbf{S}$. The preceding analysis shows how the problem of waveform design should be approached.

4.3. FH Code Optimization. In this part, we present a FH code design method, where the entries of $\mathbf{C}$ are design variables that can be optimized to achieve a desired condition number.

As the code matrix $\mathbf{C}$ is a discrete set, minimizing the object function in (21) is NP-hard. Traditional greedy optimization algorithms will easily get trapped into local optima. As a statistical optimization technique, simulated annealing (SA) algorithm [32] could find the global optimal or nearly optimal solutions. The SA algorithm runs a Markov chain Monte Carlo (MCMC) sampling on the discrete feasible set. By running the MCMC and gradually decreasing the temperature, the generated sample will have a high probability to have a small object function output. In this paper, a slightly modified form of SA called QSA [13], which allows faster convergence, is used to optimize the FH code $\mathrm{C}$. The parameters of QSA are temperature $T$, decrease rate of temperature $\alpha$, jump size $J$, and decrease rate of jump size $\beta$. QSA is initialized with values of $T>0$ and $J>0$, choosing $\alpha$ and $\beta$ from $(0,1)$. The basic steps to apply the QSA algorithm to the $\mathrm{FH}$ code matrix design are summarized as follows.

(1) Randomly draw a code matrix $\mathbf{C}$ from the set $\{0,1, \ldots, G-1\}$.

(2) Randomly draw $j$ from $\{1,2, \ldots,\lceil J\rceil\}$.

(3) Set $\mathbf{C}^{\prime}=\mathbf{C}$, and repeat the following operations $j$ times: Randomly draw $m$ from $\{1,2, \ldots, M\}$ and $q$ from $\{0,1, \ldots, Q-1\}$; select $g$ from $\{0,1, \ldots, G-1\} \backslash$ $\left\{c_{m, q}, \forall m\right\}$; set $c_{m, q}^{\prime}=g$.

(4) Compute the Gram matrix $\mathbf{S}^{H} \mathbf{S}$ from (30), and calculate the condition number $f(\mathbf{C})=\operatorname{cond}\left(\mathbf{S}^{H} \mathbf{S}\right)$.

(5) Randomly draw $U$ from $[0,1]$. If $U<\exp ((f(\mathbf{C})-$ $\left.\left.f\left(\mathbf{C}^{\prime}\right)\right) / T\right), \mathbf{C} \leftarrow \mathbf{C}^{\prime}$.

(6) Set $T \leftarrow \alpha T$ and $J \leftarrow \beta J$.

(7) If $f(\mathbf{C})$ is small enough or nearly unchanged after several iterations, terminate the algorithm. Else, go to Step (2).

\section{Simulations and Discussions}

In this section, numerical examples are conducted to demonstrate the performance improvement obtained by the proposed $\mathrm{FH}$ code optimization method for RCI with modeling error. First, an imaging scene is presented. An X-band RCI system with carrier frequency of $10 \mathrm{GHz}$ is considered. The transmitters are configured as a ULA with $M=8$ and interelement spacing $d=1 \mathrm{~m}$, respectively. The $\mathrm{FH}$ waveforms are comprised by $Q=128$ hopping frequencies with $\Delta t=10 \mathrm{~ns}, \Delta f=1 \mathrm{MHz}$, and $G=500$. A range-azimuth target space, covering $10 \mathrm{~m} \times 10 \mathrm{~m}$, is discretized to $10 \times 10$ grid-cells.

5.1. Verification of the Proposed Method. First, we consider the FH code optimization process. The decrease of the object

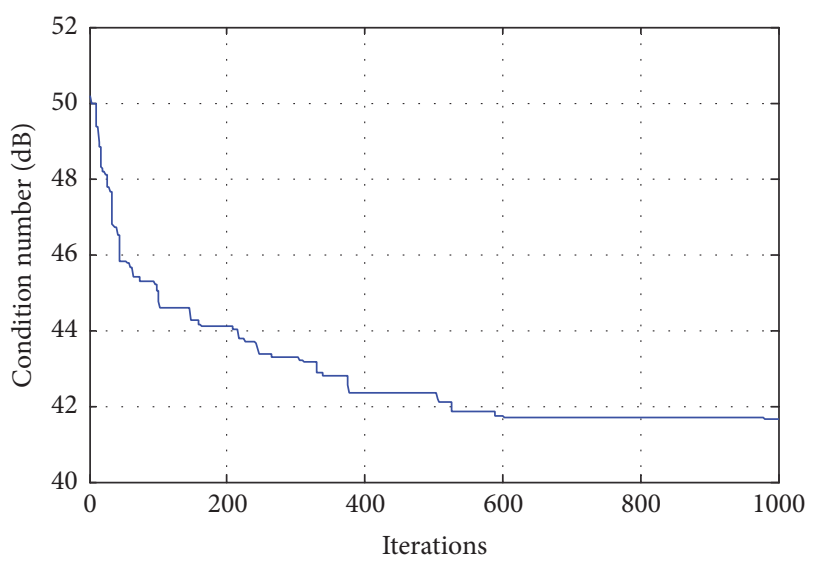

FIgURE 7: Decrease of object function versus the number of iterations.

function $f(\mathbf{C})$ with respect to the iterations of QSA is shown in Figure 7, which means that the condition number of the Gram matrix $\mathbf{S}^{H} \mathbf{S}$ is reduced during the optimization process. Besides, the decrease rate becomes slow as the iterations increase, which means the object function $f(\mathbf{C})$ converges.

Figure 8 presents the histograms of the normalized singular values $\sigma_{k} / \sigma_{\max }(k=1, \ldots, K)$ for different $\mathrm{FH}$ codes. Besides the optimized code and random code before optimization, the other two pseudorandom codes, that is, Costas code and Bernoulli code, are introduced for comparison. The random code, which is distributed uniformly in $[0, G-1]$, is the initial code for our proposed design method. Costas code [33] has the nearly ideal autocorrelation property, but the cross-correlation property for any two or more Costas codes cannot be guaranteed. Bernoulli code is a chaotic sequence generated by Bernoulli map [34], which is well orthogonal and can be flexibly and easily generated with arbitrary code length for arbitrary number of transmitters. As depicted in Figure 8, our proposed design method decreases the condition number cond $\left(\mathbf{S}^{H} \mathbf{S}\right)$ from $50.29 \mathrm{~dB}$ to $41.65 \mathrm{~dB}$ by optimizing the $\mathrm{FH}$ code. Besides, the singular values are centralized with the number of large singular values increased and the number of small singular values decreased; thus the condition number $\sigma_{\max } / \sigma_{\min }$ becomes small. Although Bernoulli code and Costas code are good candidates for FH-RCI, the condition numbers using the two codes are larger than the optimized code since they are not optimized according to the reference matrix.

Figures 9 and 10 show the auto- and cross-correlation functions for the four $\mathrm{FH}$ codes, respectively. Although there are $M=8$ transmitters, each of them emits the same type of FH waveform, for example, Costas code. Then we randomly select one of the eight transmitters to plot the autocorrelation function and two of them to plot the crosscorrelation function. As depicted in Figures 9 and 10, the four types of $\mathrm{FH}$ codes which are all randomly distributed in $[0, G-1]$ have good auto- and cross-correlation properties. As expected, the autocorrelations are very close to the Dirac delta function, and the cross-correlation functions are almost zeros. However, the random code, Costas code, and Bernoulli 


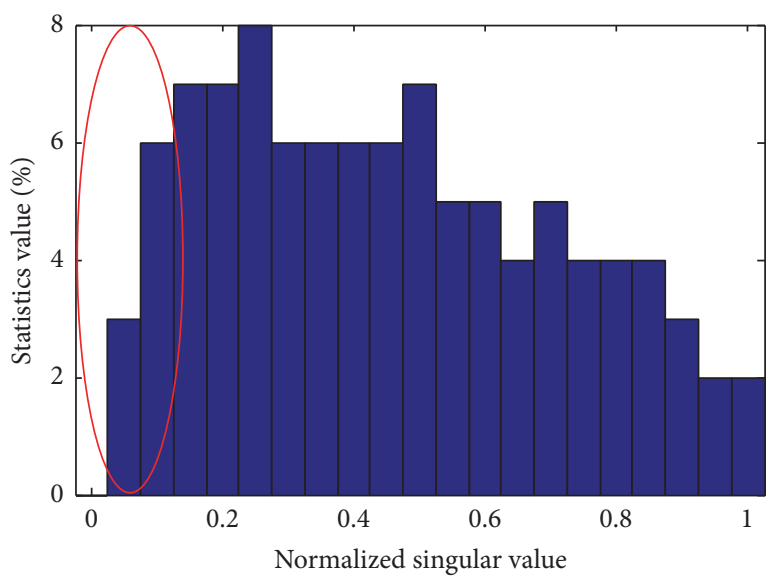

(a)

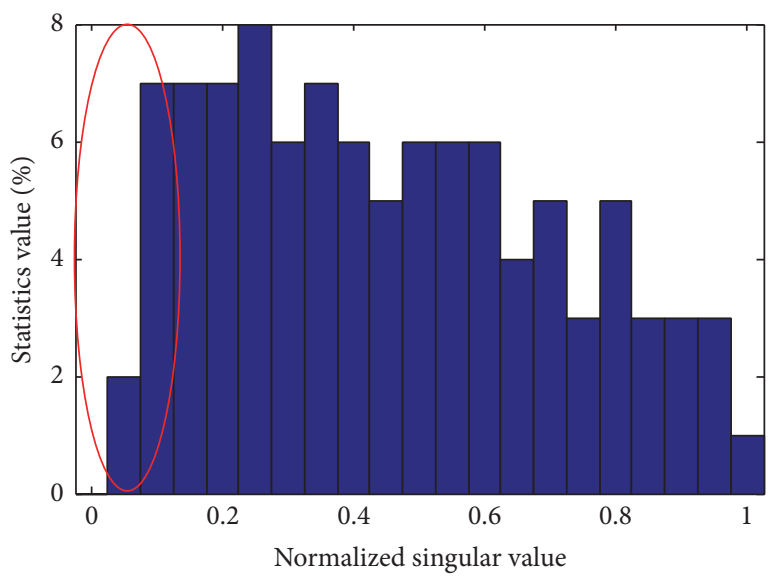

(c)

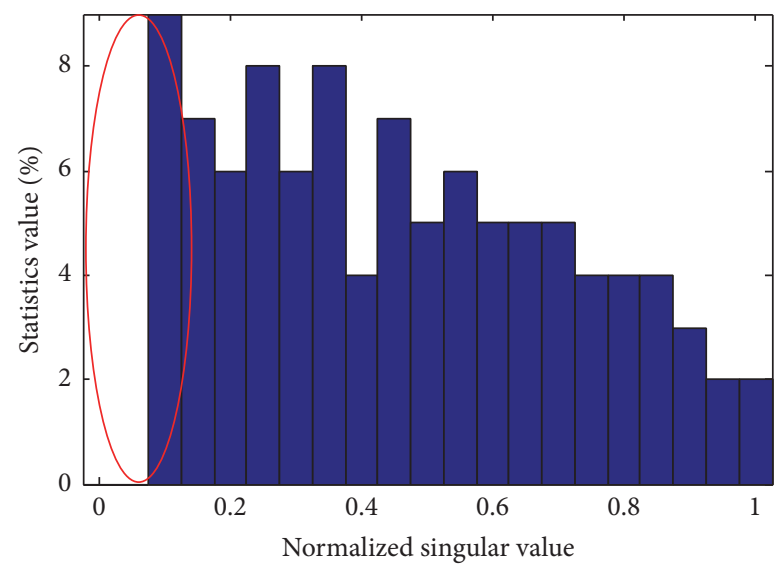

(b)

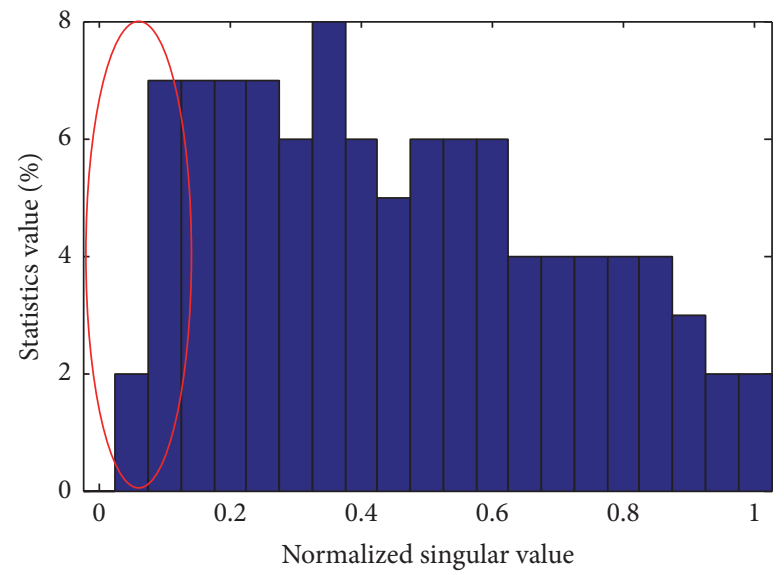

(d)

Figure 8: Histogram of the normalized singular values for different FH codes. (a) Random code, cond $\left(\mathbf{S}^{H} \mathbf{S}\right)=50.29 \mathrm{~dB}$; (b) optimized code, cond $\left(\mathbf{S}^{H} \mathbf{S}\right)=41.65 \mathrm{~dB}$; (c) Bernoulli code, $\operatorname{cond}\left(\mathbf{S}^{H} \mathbf{S}\right)=48.67 \mathrm{~dB}$; (d) Costas code, cond $\left(\mathbf{S}^{H} \mathbf{S}\right)=50.71 \mathrm{~dB}$.

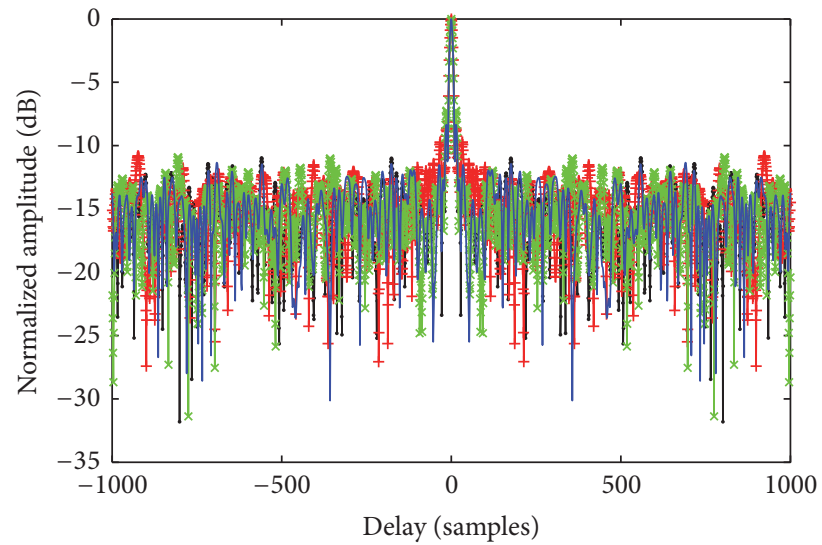

$\begin{array}{ll}- \text { Random code } & * \text { Costas code } \\ \leftarrow \text { Bernoulli code } & \quad \text { Optimized code }\end{array}$

FIGURE 9: Autocorrelation for different FH codes.

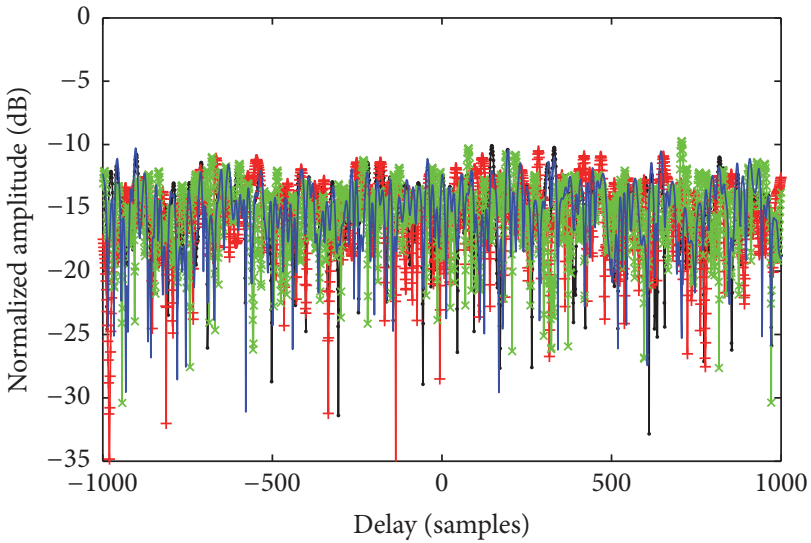

$\begin{array}{ll}\longrightarrow \text { Random code } & * \text { Costas code } \\ \longrightarrow \text { Bernoulli code } & \quad \text { Optimized code }\end{array}$

Figure 10: Cross-correlation for different FH codes. 


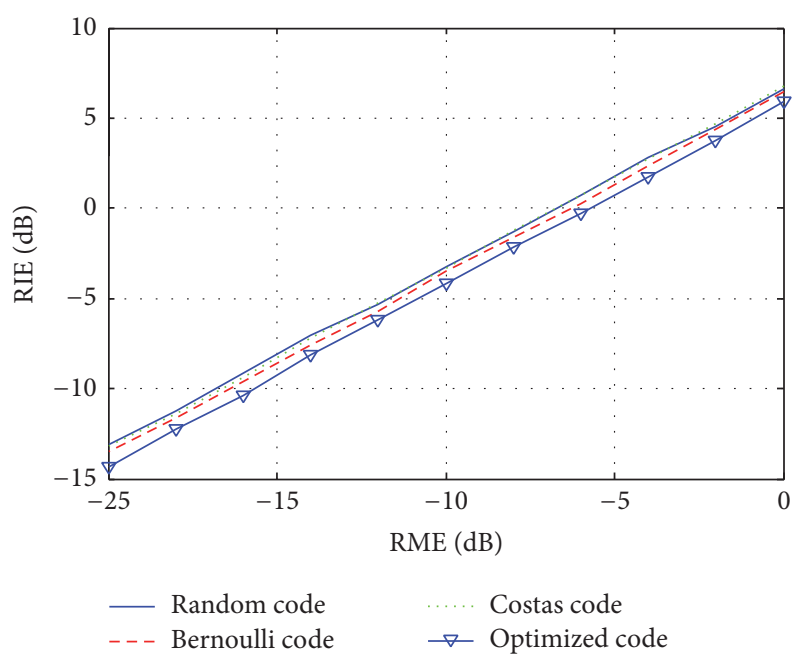

FIGURE 11: RIE as a function of RMEs for different FH codes.

code show inferior performance than the optimized code, from the perspective of condition number. Besides the autoand cross-correlation properties, other factors, for example, the grid-cell size, number of transmitters, and interelement spacing, would also impact the condition number of the Gram matrix $\mathbf{S}^{H} \mathbf{S}$. This implies that the $\mathrm{FH}$ code should be designed by considering both the $\mathrm{FH}$ waveform and the system configuration.

Next, we demonstrate the performance improvement due to the FH design by plotting the RIE as a function of RME. Figure 11 compares the curves of RIE with respect to RME for different codes, where the condition numbers of $\mathbf{S}^{H} \mathbf{S}$ for the four $\mathrm{FH}$ codes are $50.29 \mathrm{~dB}, 48.67 \mathrm{~dB}, 50.71 \mathrm{~dB}$, and $41.65 \mathrm{~dB}$, respectively. We observe that, for all RMEs, random code, Costas code, and Bernoulli code have similar performance, and the optimized code provides a marked performance improvement by about $1 \mathrm{~dB}$ of RIE. The improvement proves the effectiveness of the proposed FH code method.

5.2. Condition Number versus Code Length. In RCI, the temporal diversity of radiation field is stochastic, which brings additional information for resolving targets. Thus, we could increase the code length to gain more independent equations in (7), which would increase the temporal stochastic degree of radiation field and decrease the condition number of the Gram matrix $\mathbf{S}^{H} \mathbf{S}$. To verify the conclusion, we conduct a numerical simulation where the code length varies in $110 \leq$ $Q \leq 200$. For comparison, the condition numbers of random code, Costas code, and Bernoulli code are presented.

The results are shown in Figure 12. The random code, Bernoulli code, and Costas code have excellent properties of autocorrelation and cross-correlation, and their condition numbers under different code lengths are nearly the same as shown in Figure 12. In addition, the condition number decreases when the code length increases, which means the incoherence of the reference matrix is improved. Because the code is random, more $\mathrm{FH}$ codes would increase the

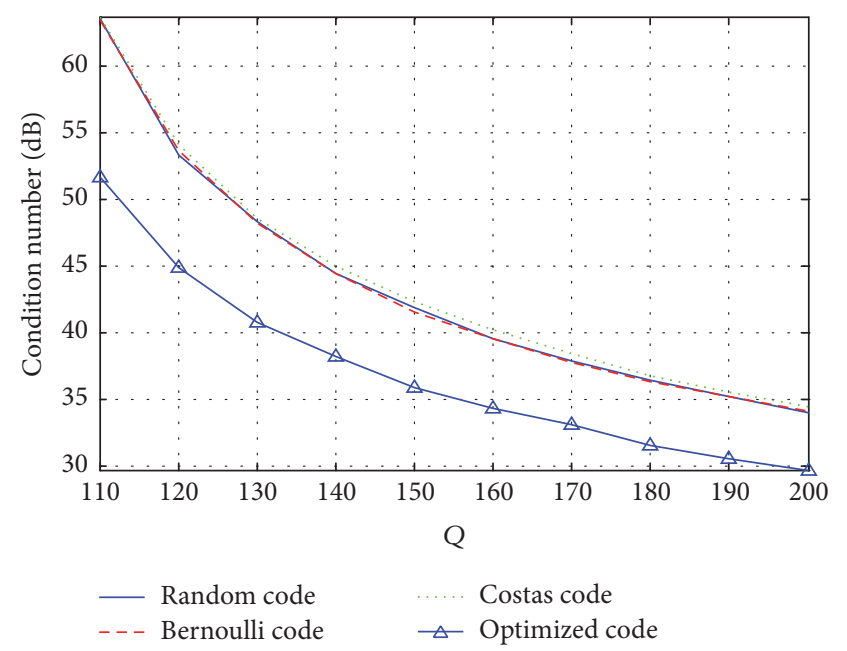

Figure 12: Condition numbers for different code lengths.

independent equations and bring more information for target reconstruction. Comparably, our proposed method can provide a code with smaller condition number than other types of code.

5.3. Condition Number versus Grid-Cell Size and Number of Transmitters. In this subsection, we consider the influence of grid-cell size and transmitter number $M$ on the condition number. As expressed in (30), the coherence of different columns of the reference matrix $\mathbf{S}$, that is, $\left\langle\mathbf{S}_{k}, \mathbf{S}_{k^{\prime}}\right\rangle$, highly depends on the grid-cell size and number of transmitters. To provide further insight into the relationships, we conduct the following experiments where the grid-cell size changes from $0.8 \mathrm{~m} \times 0.8 \mathrm{~m}, 1 \mathrm{~m} \times 1 \mathrm{~m}$ to $1.2 \mathrm{~m} \times 1.2 \mathrm{~m}$ and the number of transmitters $M$ varies from 2 to 16 .

Figure 13 shows the optimized condition number of Gram matrix by our proposed method. It can be concluded that a larger grid-cell makes the condition number smaller. As shown in (30), a larger grid-cell increases the delay difference $\tau=\tau_{m}^{k}-\tau_{m^{\prime}}^{k^{\prime}}$, which leads to the detecting signals $\mathbf{S}_{k}$ and $\mathbf{S}_{k^{\prime}}$ less coherent. Therefore, increasing the grid-cell size gains the stochastic degree of radiation field, and then the imaging performance is improved and more robust to modeling error. More transmitters emit more independent waveforms, which results in a more incoherent radiation field after being optimized by selecting an optimal FH code. However, the waveform optimizing is more time-consuming when the number of transmitters is increased. Besides, the waveform design method is less efficient, and the incoherence of $\mathbf{S}$ changes slightly after numerous QSA operations when the condition number is small enough, as shown in Figure 13. This means the FH code affects the condition number slightly in this case. Accordingly, the simulation result could be a guideline on the configuration of transmitters. On one hand, increasing the number of transmitters makes the condition number of reference matrix become small especially when the radiation field is coherent. On the other hand, employing 


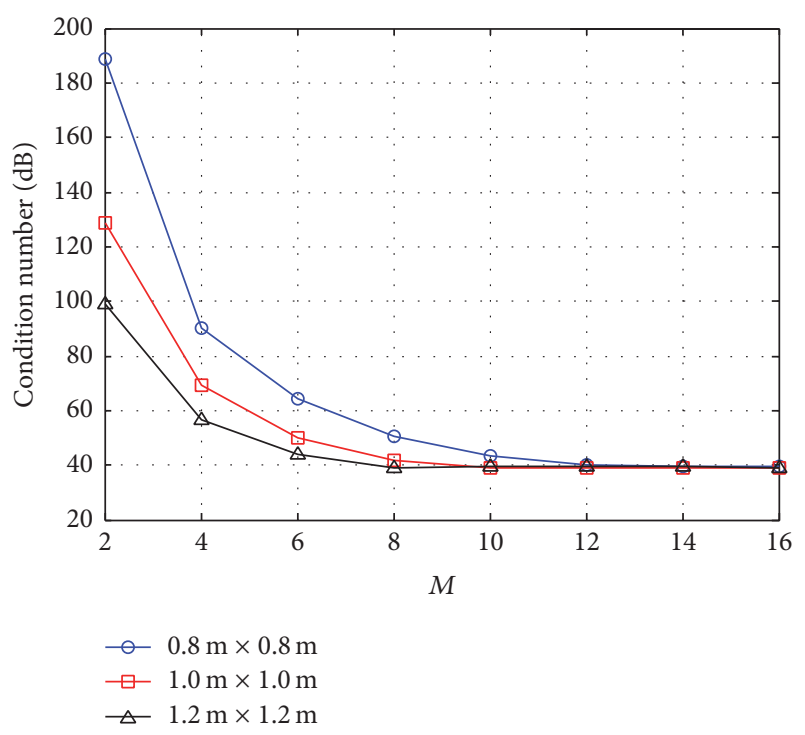

Figure 13: Condition numbers for different grid-cell sizes and transmitter number.

more transmitters further is meaningless when the condition number is small enough and changes slightly, and the hardware cost would be unacceptable in this case.

\section{Conclusion}

This paper considers the FH-RCI with modeling error and focuses on FH code design method. For FH-RCI, the transmitted FH waveforms play an important role in the shape of an incoherence reference matrix, since different waveforms could generate the detecting signals with different stochastic degrees. To increase the numerical stability of RCI with modeling error, we optimize the reference matrix by selecting the FH codes for all the transmitters. In our method, the design criterion of minimizing the condition number of the reference matrix is proposed, which is different from conventional waveform design criterions and could reduce the imaging error of LS method. Based on this principle, we first derive the analytical expression of the correlation between the different columns of the reference matrix. Then we optimally design the FH code using QSA algorithm. Results of numerical experiments show that the proposed method could generate a reference matrix with smaller condition number and improve the imaging performance of $\mathrm{RCI}$ with modeling error.

\section{Competing Interests}

The authors declare that they have no competing interests.

\section{Acknowledgments}

This work is supported by the National Natural Science Foundation of China (nos. 61302149, 61571011, and 61302142).

\section{References}

[1] D. Li, X. Li, Y. Qin, Y. Cheng, and H. Wang, "Radar coincidence imaging: an instantaneous imaging technique with stochastic signals," IEEE Transactions on Geoscience and Remote Sensing, vol. 52, no. 4, pp. 2261-2277, 2014.

[2] X. Zhou, H. Wang, Y. Cheng, and Y. Qin, "Radar coincidence imaging with phase error using Bayesian hierarchical prior modeling," Journal of Electronic Imaging, vol. 25, no. 1, Article ID 013018, 2016.

[3] C.-Y. Chen and P. P. Vaidyanathan, "MIMO radar ambiguity properties and optimization using frequency-hopping waveforms," IEEE Transactions on Signal Processing, vol. 56, no. 12, pp. 5926-5936, 2008.

[4] S. Gogineni and A. Nehorai, "Frequency-hopping code design for MIMO radar estimation using sparse modeling," IEEE Transactions on Signal Processing, vol. 60, no. 6, pp. 3022-3035, 2012.

[5] T. Huang, Y. Liu, H. Meng, and X. Wang, "Cognitive random stepped frequency radar with sparse recovery," IEEE Transactions on Aerospace and Electronic Systems, vol. 50, no. 2, pp. 858870, 2014.

[6] H. Deng, "Discrete frequency-coding waveform design for netted radar systems," IEEE Signal Processing Letters, vol. 11, no. 2, pp. 179-182, 2004.

[7] J. Li, P. Stoica, and X. Zheng, "Signal synthesis and receiver design for MIMO radar imaging," IEEE Transactions on Signal Processing, vol. 56, no. 8, pp. 3959-3968, 2008.

[8] A. Guruswamy and R. Blum, "Ambiguity optimization for frequency-hopping waveforms in MIMO radars with arbitrary antenna separations," IEEE Signal Processing Letters, vol. 23, no. 9, pp. 1231-1235, 2016.

[9] J. Li, L. Xu, P. Stoica, K. W. Forsythe, and D. W. Bliss, "Range compression and waveform optimization for MIMO radar: a Cramér-Rao bound based study," IEEE Transactions on Signal Processing, vol. 56, no. 1, pp. 218-232, 2008.

[10] D. R. Fuhrmann and G. San Antonio, "Transmit beamforming for MIMO radar systems using signal cross-correlation," IEEE Transactions on Aerospace and Electronic Systems, vol. 44, no. 1, pp. 171-186, 2008.

[11] P. Stoica, J. Li, and Y. Xie, "On probing signal design for MIMO radar," IEEE Transactions on Signal Processing, vol. 55, no. 8, pp. 4151-4161, 2007.

[12] L. Wang, K.-K. Wong, H. Wang, and Y. Qin, "MIMO radar adaptive waveform design for extended target recognition," International Journal of Distributed Sensor Networks, vol. 2015, Article ID 154931, 11 pages, 2015.

[13] S. Badrinath, A. Srinivas, and V. U. Reddy, "Low-complexity design of frequency-hopping codes for MIMO radar for arbitrary Doppler," EURASIP Journal on Advances in Signal Processing, vol. 2010, Article ID 319065, 2010.

[14] X. Zhou, H. Wang, Y. Cheng, and Y. Qin, "Sparse autocalibration for radar coincidence imaging with gain-phase errors," Sensors, vol. 15, no. 11, pp. 27611-27624, 2015.

[15] X. Zhou, H. Wang, Y. Cheng, Y. Qin, and H. Chen, "Radar coincidence imaging for off-grid target using frequency-hopping waveforms," International Journal of Antennas and Propagation, vol. 2016, Article ID 8523143, 16 pages, 2016.

[16] X. Zhou, H. Wang, Y. Cheng, and Y. Qin, "Off-grid radar coincidence imaging based on variational sparse bayesian learning," 
Mathematical Problems in Engineering, vol. 2016, Article ID 1782178, 12 pages, 2016.

[17] C.-Y. Chen and P. P. Vaidyanathan, "Compressed sensing in MIMO radar," in Proceedings of the 42nd Asilomar Conference on Signals, Systems and Computers (ASILOMAR '08), pp. 41-44, Pacific Grove, Calif, USA, October 2008.

[18] N. S. Subotic, B. Thelen, K. Cooper et al., "Distributed RADAR waveform design based on compressive sensing considerations," in Proceedings of the IEEE Radar Conference (RADAR '08), Rome, Italy, May 2008.

[19] Y. Yu, S. Sun, R. N. Madan, and A. Petropulu, "Power allocation and waveform design for the compressive sensing based MIMO radar," IEEE Transactions on Aerospace and Electronic Systems, vol. 50, no. 2, pp. 898-909, 2014.

[20] J. Zhang, D. Zhu, and G. Zhang, "Adaptive compressed sensing radar oriented toward cognitive detection in dynamic sparse target scene," IEEE Transactions on Signal Processing, vol. 60, no. 4, pp. 1718-1729, 2012.

[21] K. Han and A. Nehorai, "Jointly optimal design for MIMO radar frequency-hopping waveforms using game theory," IEEE Transactions on Aerospace and Electronic Systems, vol. 52, no. 2, pp. 809-820, 2016.

[22] D. Li, X. Li, Y. Cheng, Y. Qin, and H. Wang, "Radar coincidence imaging under grid mismatch," ISRN Signal Processing, vol. 2014, Article ID 987803, 8 pages, 2014.

[23] X. Xu, X. Zhou, Y. Cheng, and Y. Qin, "Radar coincidence imaging with array position error," in Proceedings of the 5th IEEE International Conference on Signal Processing, Communications and Computing (ICSPCC '15), Ningbo, China, September 2015.

[24] Y. Chi, L. L. Scharf, A. Pezeshki, and A. R. Calderbank, "Sensitivity to basis mismatch in compressed sensing," IEEE Transactions on Signal Processing, vol. 59, no. 5, pp. 2182-2195, 2011.

[25] H. Zhu, G. Leus, and G. B. Giannakis, "Sparsity-cognizant total least-squares for perturbed compressive sampling," IEEE Transactions on Signal Processing, vol. 59, no. 5, pp. 2002-2016, 2011.

[26] G. H. Golub and C. F. Van Loan, Matrix computations, Johns Hopkins Studies in the Mathematical Sciences, Johns Hopkins University Press, Baltimore, MD, USA, Third edition, 1996.

[27] Z. Yang, C. Zhang, and L. Xie, "Robustly stable signal recovery in compressed sensing with structured matrix perturbation," IEEE Transactions on Signal Processing, vol. 60, no. 9, pp. 46584671, 2012.

[28] A. N. Tikhonov, "Solution of incorrectly formulated problems and the regularization method," Soviet Mathematics Doklady, vol. 4, pp. 1035-1038, 1963.

[29] D. L. Phillips, "A technique for the numerical solution of certain integral equations of the first kind," Journal of the Association for Computing Machinery, vol. 9, pp. 84-97, 1962.

[30] M. Çetin and W. C. Karl, "Feature-enhanced synthetic aperture radar image formation based on nonquadratic regularization," IEEE Transactions on Image Processing, vol. 10, no. 4, pp. 623631, 2001.

[31] J. Tong, Q. Guo, S. Tong, J. Xi, and Y. Yu, "Condition numberconstrained matrix approximation with applications to signal estimation in communication systems," IEEE Signal Processing Letters, vol. 21, no. 8, pp. 990-993, 2014.

[32] S. Kirkpatrick, J. Gelatt, and M. P. Vecchi, "Optimization by simulated annealing," American Association for the Advancement of Science. Science, vol. 220, no. 4598, pp. 671-680, 1983.
[33] J. P. Costas, "A study of a class of detection waveforms having nearly ideal range-doppler ambiguity properties," Proceedings of the IEEE, vol. 72, no. 8, pp. 996-1009, 1984.

[34] Y. Jin, H. Wang, W. Jiang, and Z. Zhuang, "Complementarybased chaotic phase-coded waveforms design for MIMO radar," IET Radar, Sonar and Navigation, vol. 7, no. 4, pp. 371-382, 2013. 


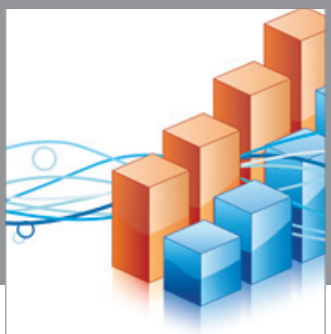

Advances in

Operations Research

vatem alat4

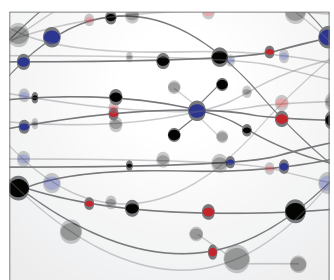

\section{The Scientific} World Journal
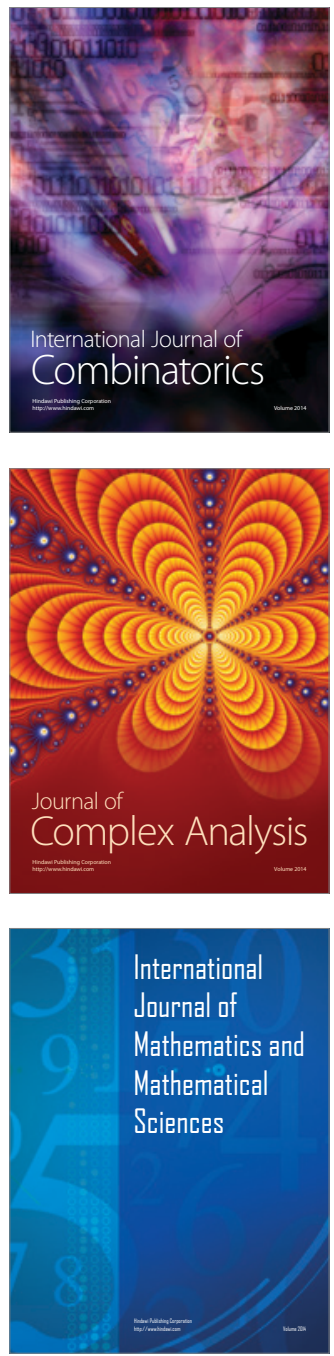
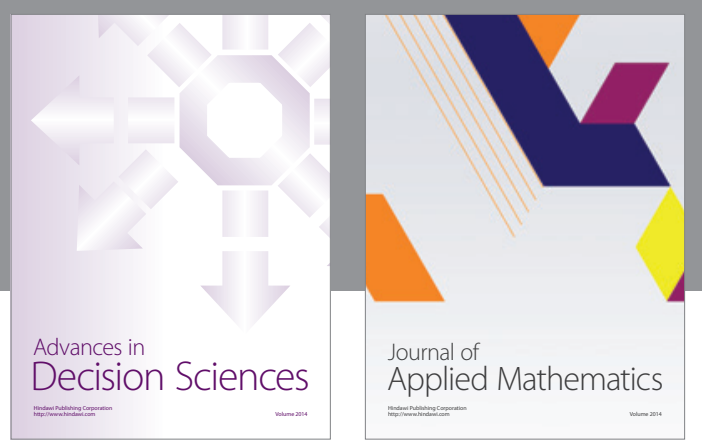

Algebra

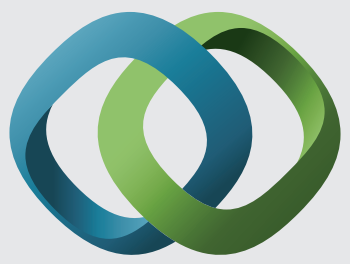

\section{Hindawi}

Submit your manuscripts at

https://www.hindawi.com
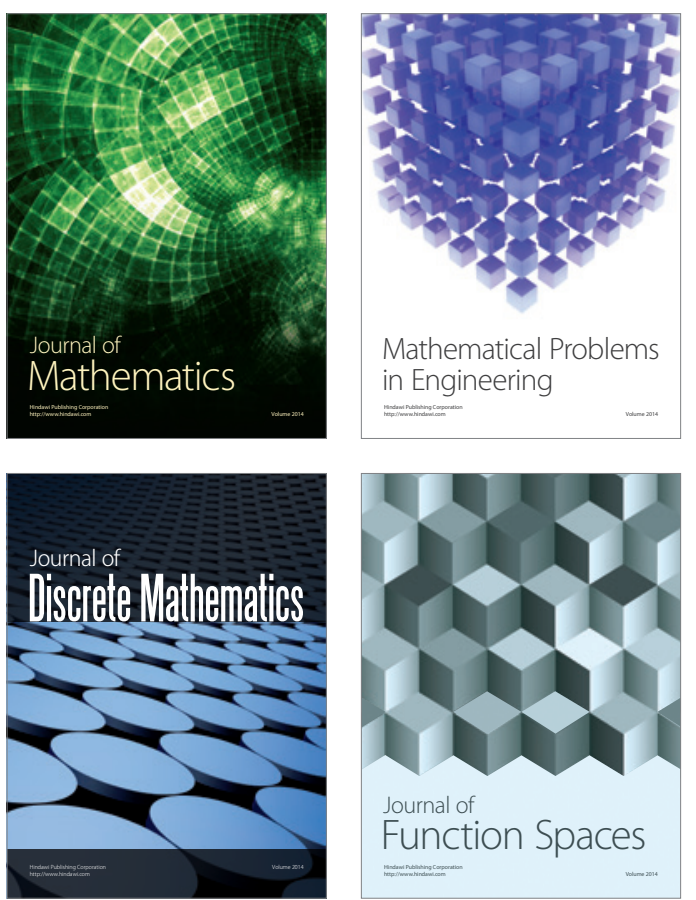

Mathematical Problems in Engineering
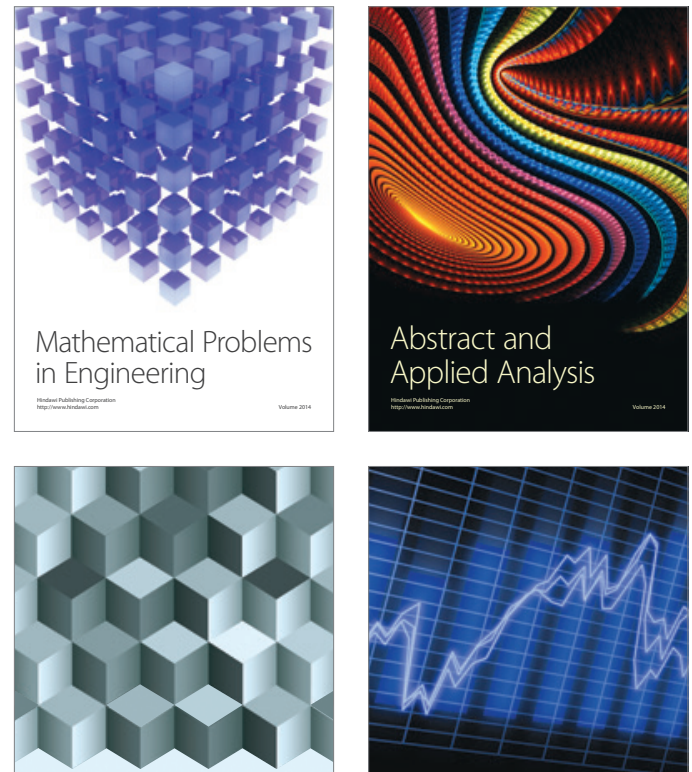

Journal of

Function Spaces

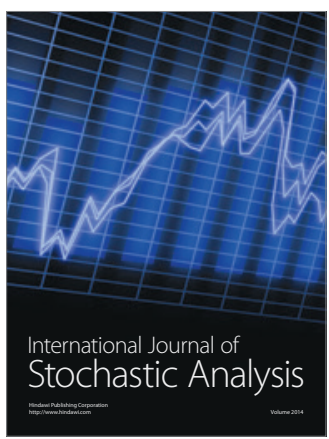

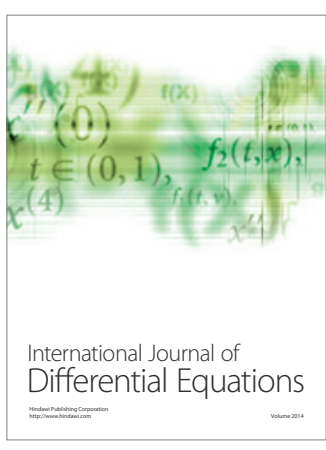
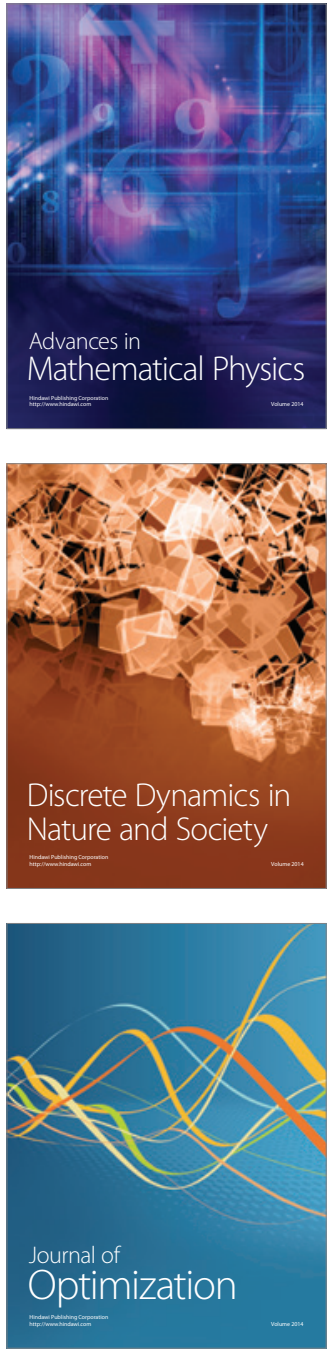